\title{
SPATIAL DAMAGE DETECTION IN ELECTRICALLY ANISOTROPIC FIBER- REINFORCED COMPOSITES USING CARBON NANOTUBE NETWORKS
}

\author{
Gerard J. Gallo and Erik T. Thostenson*1 \\ Department of Mechanical Engineering and Center for Composite Materials \\ University of Delaware \\ Newark, DE 19716 USA
}

\begin{abstract}
Due to their small size, carbon nanotubes are able to create electrically conductive networks surrounding structural fiber reinforcements in composites. As damage accumulates in the polymer matrix, cracks break the nerve-like conducting pathways resulting in bulk changes in electrical resistance. To utilize carbon nanotube networks as a potential structural health monitoring (SHM) approach it is necessary to develop techniques capable of detecting localized and site-specific damage. Electrically anisotropic composites were manufactured from unidirectional glass fibers treated with a sizing containing carbon nanotubes. Local damage was introduced to the composite laminates and was assessed by taking electrical measurements from an array of surface mounted electrodes. Conductivity maps generated using electrical impedance tomography (EIT) were compared to the normalized resistance change approach. A center of mass approach was utilized to define the damage location. Both techniques are capable of localizing damage in the composite but EIT has much higher overall spatial sensitivity. The normalized resistance change approach, however, requires significantly fewer measurements and can be utilized for real-time detection of damage.
\end{abstract}

\footnotetext{
Keywords: Damage sensing, carbon nanotubes, electrical impedance tomography

* Corresponding author: Fax: +1 3028313619

E-mail address: thosten@udel.edu (Erik Thostenson) 


\section{Introduction}

Advanced polymer composites are increasingly being used in structural applications because of their desirable properties, such as improved corrosion resistance [1], high strength-toweight ratios [2] and increased fatigue performance [3] as compared to traditional materials. However, structural failure in composites can be difficult to predict, and subsurface micro-scale cracks initiate in the polymer matrix. Structural health monitoring (SHM) is increasingly necessary to maintain structural integrity, improve safety and reliability, and to reduce maintenance costs. SHM provides diagnostic information on the magnitude and location of damage. A variety of SHM systems have been developed utilizing strain gages [4], fiber optics [5], [6], and acoustic sensors [7]. There is increasing interest in developing advanced SHM techniques that can sense large areas, are minimally invasive, and capable of real-time sensing.

It is well established that the electrical properties of carbon fiber composites can be utilized to detect damage. Schulte et al. [8] first demonstrated this approach by attaching electrodes to ends of a carbon fiber/epoxy laminate and monitoring the resistance changes under tensile loading. Fiber fracture resulted in permanent changes in electrical conductivity. Thostenson and Chou $[9,10]$ extended electrical damage sensing to glass fiber composites by utilizing carbon nanotubes dispersed in the polymer matrix as the electrical network. They showed nanotube networks were particularly sensitive to detecting the onset and accumulation of matrix cracking.

Several researchers have worked to advance the electrical resistance approach for damage sensing into an SHM technique capable of locating damage in large-scale structures. Todoroki et al. $[11,12]$ applied the response surface methodology to approximate the location of delamination in carbon fiber composites. Using this statistical technique, they performed one and 
two-dimensional spatial analysis. However, accurate predications required electrodes to be placed over the entire surface. Angelidis and co-workers [13] proposed a less invasive procedure, referred to as the potential method, where electrodes are placed only at the boundary. As current is sourced between electrode pairs the potential is measured at each electrode and damaged areas approximated by analyzing changes in the potential distribution.

Electrical impedance tomography (EIT) is an imaging technique that reconstructs spatial conductivity from a set of boundary measurements. Similar to the potential method, current injection and voltage measurements are acquired from electrodes around the boundary. Schueler and co-workers [14] first suggested the application of EIT in fiber composites for damage mapping. However, at the time of the work reconstruction algorithms had not been developed to account for electrical anisotropy. Hou and Loh [17, 18] applied the technique to composite structures by generating conductivity reconstructions of electrically isotropic carbon nanotube sheets. Through their work, they demonstrated the ability to locate inhomogeneous regions, map strain fields, and distinguish between multiple regions of damage. Baltopoulous and et al. [17] applied the EIT technique to carbon fiber composites. The high electrical anisotropy was mitigated by using a woven layup and assuming the laminate to be electrically quasi-isotropic. However, the quality of the generated conductivity images was poor, and additional postprocessing was required to accurately approximate the damage location. Improvements in reconstruction algorithms allowed Loyola et al. [18] to extend EIT to electrically orthotropic composites. Measurements were obtained from a thin multi-walled carbon nanotube film on the surface of a unidirectional glass fiber composite (planar conductivity ratio of 1:2). The study served as an introduction of EIT in orthotropic composites and examined the accuracy of the method in terms of detecting and locating various forms of surface damage. One disadvantage of 
EIT is the time associated with the construction of the electrical conductivity map, making realtime monitoring difficult.

In this paper, we assess localized damage in electrically anisotropic composites using normalized resistance measurements and conductivity maps generated using EIT. The two techniques are compared in terms of real-time application and accurate characterization of the damage site. Glass fibers treated with a sizing containing dispersed carbon nanotubes are used to produce composites with a planar conductivity ratio of 1:5. Holes are drilled into the laminates to represent local matrix and fiber damage, and delamination and matrix cracking is created from drop-weight impact. Three different current injection patterns are introduced for acquiring measurements. Electrical resistance measurements are obtained from a network of electrodes before and after damage. Differential voltage measurements are also collected from the electrode network in order to generate conductivity reconstructions using EIT. Center of mass equations are modified in order to approximate the damage location from the normalized resistance measurements and conductivity reconstructions, and the accuracy of each technique is compared in light of the number of measurements required.

\section{Experimental Methods and Data Analysis}

\subsection{Materials and Composites Manufacturing}

All laminates used in this study were manufactured using the same technique for conductively modifying the fibers with nanotubes and consolidation of the final composite. Unidirectional E-glass nonwoven fabric (Jamestown Distributers) was treated with a fiber sizing containing dispersed multi-walled carbon nanotubes (SIZICYL XC R2G, solid content of 6.2\%). The glass fabric also has an epoxy compatible sizing. 
The sizing was applied using modified vacuum bag infusion. Illustrated in Figure 1, the sized fibers were placed on top of a high permeability layer with peel-ply on either side and then sealed with an injection line by a semi-permeable membrane (Dalhtex SP-2, Airtech). The membrane traps the solid content while allowing liquid to evaporate. The assembly was sealed in a vacuum bag and placed under vacuum. The sizing was then drawn into the fibers under vacuum. To assure that the sizing is homogeneous prior to infusion it was first mixed in a centrifugal mixer (ARE-310, THINKY) at 2000 RPM for 30 seconds and then placed in an ultrasonic bath (Branson 1510) for 15 minutes. Once the fibers were fully infused they were dried under vacuum at $150^{\circ} \mathrm{C}$ for 4 hours [19]. The deposition of nanotubes and other solids from the sizing results in a mass increase of approximately $1 \%$ [20].

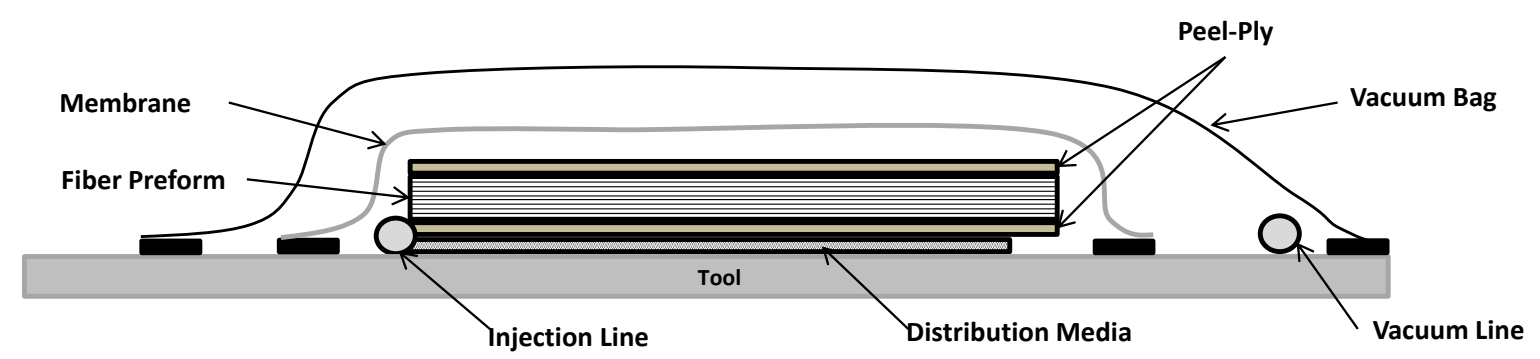

Figure 1: Illustration of the modified vacuum bag setup used for sizing fibers with nanotubes.

Composites were manufactured using vacuum assisted resin transfer molding (VARTM). Before infusion, the two-part epoxy resin (EPON 862, 862/Epi-Kure W, 100:26.4, Momentive Specialty Chemicals, Inc.) was de-gassed in a vacuum oven for 30 minutes at $60^{\circ} \mathrm{C}$. Once infused, the composites were cured at $130^{\circ} \mathrm{C}$ for 6 hours. Square, single-ply laminae measuring 
$10.2 \times 10.2 \mathrm{~cm}$ were manufactured for the site-specific damage study. Cross-ply [0/90]s laminates measuring $8.9 \times 17.8 \mathrm{~cm}$ were manufactured for impact testing. Unidirectional $[0]_{5}$ laminates measuring $20.3 \times 5.1 \mathrm{~cm}$ were manufactured to examine delamination under tensile loading. To promote delamination, the center ply in the laminate was cut in half, transverse to the fiber direction to create a ply discontinuity where delamination would initiate under tensile loading due to shear stresses at the ply ends.

\subsection{Electrode Placement}

Prior to infusion of the epoxy, high temperature masking tape (3M) was placed at specific locations on the top layer to impede resin flow in the regions where electrodes would be placed and make it easier to expose the conductive network after infusion. The electrode placement geometry is shown in Figure 2. For each specimen 32 electrodes were attached equidistantly around the boundary. The tape was then removed after the curing. The electrode regions were polished using 320 and 400 grit sandpaper. Silver paint (04999-AB, SPI Supplies) was applied to the surface to ensure a uniform current density at the electrode. Copper wires were then bonded to the electrodes using a silver epoxy (40-3900, Epoxies, etc.). 


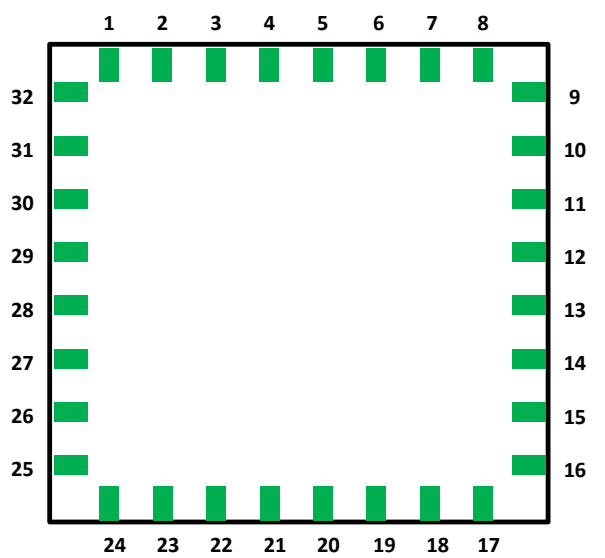

(a)

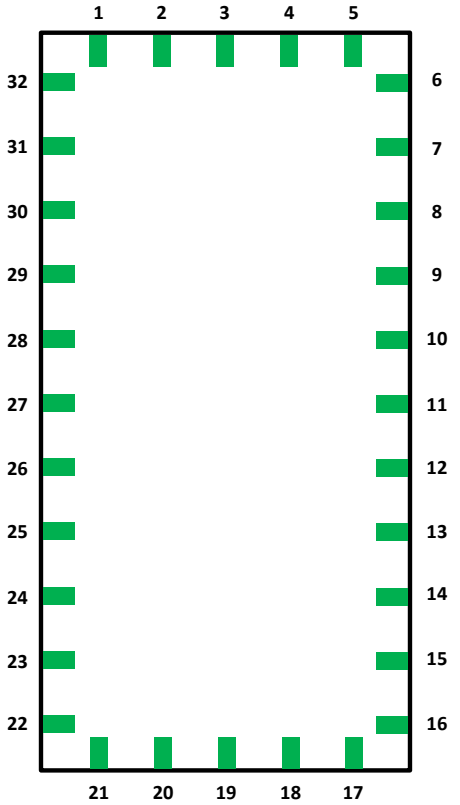

(b)

Figure 2: Illustration of tape placement for electrode preparation in (a) laminates used for site-specific testing and (b) laminates used for impact testing.

\subsection{Electrical and Mechanical Measurements}

A constant source current of $10 \mathrm{~mA}$ was applied between electrode pairs using a Keithley 6430 meter and the voltage was measured using a Keithley 2182A Nanovoltmeter. For switching the current source and voltage measurements between electrodes a Keithley 3706A switch meter was used. The measurements were synchronized using a customized LabVIEW program.

The in-plane conductivity was determined from a separate study [21]. Table 1 shows the longitudinal $\left(\sigma_{0}\right)$ and transverse $\left(\sigma_{90}\right)$ conductivity for each composite. The ratio between longitudinal and transverse conductivity is roughly 5:1. The sizing treatment creates more conducting pathways along the length of the fiber, resulting in higher longitudinal conductivity. The cross-ply conductivity was determined by assuming the plies act as resistors in parallel. 
Table 1: In-plane conductivity for E-Glass/EPON 862/nanotube composites.

\begin{tabular}{ccc}
\hline $\boldsymbol{\sigma}_{\mathbf{0}}[\mathbf{S} / \mathbf{m}]$ & $\boldsymbol{\sigma}_{\mathbf{9 0}}[\mathbf{S} / \mathbf{m}]$ & $\boldsymbol{\sigma}_{\mathbf{0} / \mathbf{9}}[\mathbf{S} / \mathbf{m}]$ \\
\hline \hline 3.89 & $7.62 \times 10^{-1}$ & 2.33 \\
\hline
\end{tabular}

To simulate local fiber and matrix damage, $1.27 \mathrm{~cm}$ diameter holes were drilled through the laminates. In one laminate, the hole was placed at the center and in the other a hole was drilled towards a corner. The area of the hole is roughly $1 \%$ of the total laminate area. Lowvelocity impact was performed on laminates in order to characterize the spatial sensitivity to localized sub-surface damage in the form of matrix cracks and ply delamination. Impact testing was performed using a drop tower equipped with a $1.27 \mathrm{~cm}$ diameter hemispherical tup (INSTRON Dynatup 8200). Impact energies were chosen in order to simulate a hand tool, such as a wrench, being dropped on the composite surface. Ultrasonic C-scans were performed postimpact to examine the damaged area. Cyclic testing was also performed on composite specimens with a ply discontinuity in order to monitor ply delamination. The test was performed using a screw-driven load frame (INSTRON 5985) at a displacement rate of $1.27 \mathrm{~mm} / \mathrm{min}$, and the load was incrementally increased by $8900 \mathrm{~N}$ following each load/unload cycle. Electrical measurements were acquired during the unloaded state following each load cycle.

\subsection{Tomographic Imaging Approach}

Electrical impedance tomography is a technique that maps the spatial electrical conductivity of a domain based on electrical measurements made at the boundary. The conductivity reconstruction is calculated using a two-step process consisting of forward and inverse problems, described below. 


\subsubsection{Forward Problem}

The forward problem solves for domain electrical potential using an initial conductivity approximation. In this study, the finite element method is applied to solve the forward problem. The governing equation is the two-dimensional form of Laplace's equation (Equation 1), where $\sigma$ is the conductivity, $\varphi$ is the electrical potential, and $I$ is the current.

$$
\nabla[\sigma(x, y) \nabla \varphi(x, y)]=-\mathrm{I}(\mathrm{x}, \mathrm{y})
$$

It is assumed that no current is generated within the domain, so the right side of Equation 1 is set to zero. For an orthotropic conductivity distribution the conductivity forms a $2 \mathrm{D}$ diagonal matrix (Equation 2) with the transverse and longitudinal conductivity along the diagonal [18].

$$
\sigma=\left[\begin{array}{cc}
\sigma_{90} & 0 \\
0 & \sigma_{0}
\end{array}\right]
$$

Boundary conditions are applied using the complete electrode model (CEM). Current injection is modeled using a Neumann boundary condition (Equation 3), where current, $I$ is injected normal to the electrode boundary, where $\mathbf{n}$ is the outward unit normal vector, and the number of electrodes is $L$. The current density along the boundary between each electrode is zero. A Dirichlet boundary condition (Equation 4) requires the voltage at each electrode to be constant and defined as the sum of the potential at the electrode and the voltage drop due to contact resistance $(z)$ between the electrode and the domain.

$$
\begin{gathered}
\int_{E_{l}} \sigma \frac{\partial \varphi}{\partial \boldsymbol{n}} d s=I_{l} \quad l=1,2, \ldots, L \\
\varphi+z_{l} \frac{\partial \varphi}{\partial \boldsymbol{n}}=V_{l} \quad l=1,2, \ldots, L
\end{gathered}
$$

To solve Equation 1, the domain is discretized using a triangular mesh with $N$ vertices. The potential is approximated as the linear sum of the potentials at each element vertex multiplied by $1^{\text {st }}$ order polynomial shape functions, $w$ (Equation 5).

$$
\varphi_{F E M}=\sum_{i=1}^{N} \varphi_{i} w_{i}
$$


The result is a linear system of equations (Equation 6). The system matrix used to solve Equation 1 is provided by $A_{M}$ while the CEM boundary conditions are implemented by matrices $A_{Z}, A_{W}$, and $A_{D}$ [22]. $A_{Z}$ and $A_{W}$ account for the effect of contact impedance on nodes underneath the electrodes, while $A_{D}$ holds the contact impedance approximation for each electrode.

$$
\left[\begin{array}{cc}
A_{M}+A_{Z} & A_{W} \\
A_{W}^{T} & A_{D}
\end{array}\right]\left\{\begin{array}{l}
\varphi \\
V
\end{array}\right\}=\left\{\begin{array}{l}
0 \\
I
\end{array}\right\}
$$

Here, $\varphi$ is a column vector containing the nodal potential distribution, while $V$ contains the electrode voltage. Current magnitudes in $I$ are assigned to both the current source and current sink electrodes while the remaining rows are assigned a magnitude of zero.

\subsubsection{Inverse Problem}

The inverse problem is driven by the Jacobian matrix which relates changes in internal conductivity to voltage at the electrodes. Equation 7 describes the two-dimensional form of the Jacobian for a given element $k$ in an electrically anisotropic medium [23]. The equation is defined as the tensor product of the gradient of the measurement potential distribution $\varphi$, orthotropic conductivity matrix $\sigma$, and the gradient of the current potential distribution $\varphi^{*}$.

$$
J^{k}=-\int \sum_{i=1}^{2} \sum_{i=1}^{2}(\nabla \varphi)_{i}(\sigma)_{i j}\left(\nabla \varphi^{*}\right)_{j}
$$

The measurement potential distribution, $\varphi$, corresponds to the nodal potential distribution obtained by solving the forward problem, described in Section 3.1. The current potential distribution, $\varphi^{*}$, is obtained by solving the forward problem using the measurement electrodes as the current injection electrodes. The inverse problem applies the Jacobian matrix along with experimentally acquired electrode potential measurements to reconstruct the conductivity distribution. 
Reconstructing the electrical conductivity distribution using EIT is a non-linear and illposed problem. Non-linearity can arise from measurement and modeling errors such as low measurement precision of the voltmeter, inaccurate approximations of electrode contact impedance, and inaccurate modeling of geometry and electrode positions relative to the test specimen. The problem is ill-posed because the solution is discontinuous, in that large local conductivity changes may correspond to very small voltage changes at the boundary [24].

In this study, the non-linearity of the EIT problem is alleviated by using a linearized onestep algorithm known as the maximum a posterior (MAP) algorithm (Equation 8).

$$
\left\{\frac{\Delta \sigma}{\sigma_{0}}\right\}=\left[\left(J^{T} W J+\lambda R\right)^{-1} J^{T} W\right]\left\{\frac{\Delta V}{V_{0}}\right\}=B\left\{\frac{\Delta V}{V_{0}}\right\}
$$

The algorithm computes normalized change in electrical conductivity based on normalized difference in potential distribution before and after a conductivity change (damage) has occurred. Due to the normalization of the voltage measurements, the Jacobian, $J$, is also normalized by the column vector of voltages calculated from the forward problem [23].

The linearized problem assumes the current magnitude, contact resistance at the electrodes, and electrode spacing remain constant. Therefore, errors related to these parameters do not affect the reconstruction. However, a disadvantage to using a non-iterative linearized method is that the quantitative result is only considered valid for small changes in conductivity and provides a largely qualitative assessment of the damage state for large-scale damage [25].

The covariance matrix, $W$, is a diagonal matrix containing the inverse of the variance for each measurement obtained using a specified current injection pattern. The matrix is used to reduce the effect of erroneous measurements with large noise [26]. In this study, a single reading was obtained for each voltage measurement and each measurement was assumed to have equal 
noise. Therefore, the covariance matrix was replaced with the identity matrix. This assumption results in a slight over approximation of the damage area.

Regularization is implemented in order to reduce the effect of modeling errors on the final solution. The technique is implemented in Equation 9 by an additional quantity, referred to as a penalty term, consisting of a regularization matrix, $R$, and smoothing parameter, $\lambda$. One of the most common methods used in EIT is Tikhonov regularization [27], which is implemented by using the identity matrix for $\mathrm{R}$. The technique increases the amplitude of each element by a fixed amount specified by the smoothing parameter. Smoothing of the solution is achieved as small values are enhanced while the elements with large magnitudes remain relatively unchanged.

The smoothing parameter in this work follows a method implemented by Adler et al. [26] where the reconstruction matrix, defined by $B$ in Equation 8, represents a signal receiver in a communications system. The output 'signal' from the receiver is the calculated conductivity change, denoted by the reconstruction matrix multiplied by the voltage measurements. The input 'signal' is the voltage measurements with corresponding measurement noise. The noise figure is defined signal-to-noise ratio of the input signal divided by the signal-to-noise ratio of the output signal. The purpose of a noise figure is to characterize a component of a network by determining how much the signal quality degrades [28]. A value for $\lambda$ is sought in Equation 9 in order to make the noise figure for the signal receiver equal to 1 . A noise figure of 1 was implemented in order to prevent over-smoothing the conductivity reconstruction.

$$
N F=\frac{S N R_{\text {in }}}{S N R_{\text {out }}}=\frac{\left(1^{t} z\right) \sqrt{N \cdot \operatorname{trace}\left(A B W^{-1} B^{t} A\right)}}{\left(1^{t} A B z\right) \sqrt{M \cdot \operatorname{trace}\left(W^{-1}\right)}}
$$


The variables $N, M$, and $A$ refer to the number of elements, number of differential voltage measurements, and area of each element, respectively. The normalized difference voltage measurements are specified by $z$.

A MATLAB script was generated to handle the EIT calculations. The script allows users to define a mesh density, electrode number and length, and the in-plane and transverse initial conductivities. The script calls functions to discretize the domain, calculate the forward problem, assemble the Jacobian, load the experimental voltage data, and calculate the conductivity change using the inverse problem. The two-dimensional mesh was generated using Distmesh [29], and EIT calculations were based on the open source EIDORS code [30]. The functions used to calculate the forward problem and Jacobian matrix were modified to include an orthogonal conductivity matrix.

\subsection{EIT Current Injection Patterns}

Three different source current injection patterns were used, illustrated in Figure 3. The red lines indicate the electrode pairs used for current injection. For each current injection pair, a set of differential voltage measurements was obtained around the boundary. Electrodes corresponding to the current source, current sink, and the ground electrode are omitted from the voltage measurements. The ground electrode is also removed from the current source assignments.

The adjacent method is commonly used in EIT applications [32, 33]. The adjacent method consists of sourcing current between neighboring electrodes, and the pattern has been shown to be more sensitive to boundary defects in isotropic media [33]. The across and diagonal patterns are implemented to provide sensitivity over the entire material surface. The across pattern consists of injecting current between electrode pairs positioned horizontally or vertically 
across the domain while in the diagonal pattern current is injected between corresponding electrodes on an adjacent side. Several studies [20, 35], have shown that electrical anisotropy can lead to differential voltage measurements of zero at the boundary, which can result in an undefined solution to the inverse problem. The diagonal pattern is advantageous for the fiber stacking sequences used in this study because it promotes current spreading for all electrode pairs.

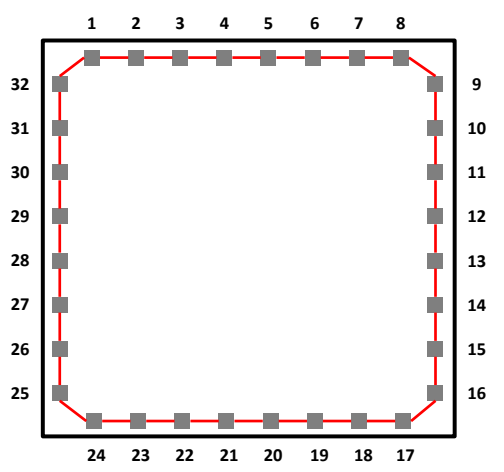

(a)

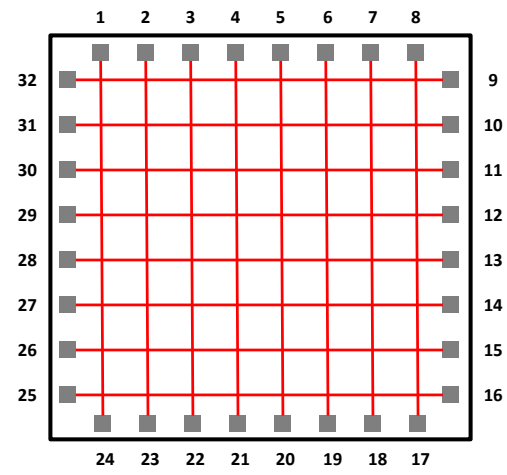

(b)

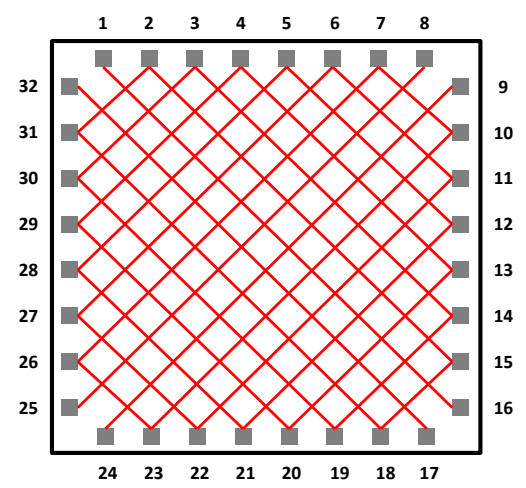

(c)

Figure 3: Illustrations of the (a) adjacent, (b) across, and (c) diagonal current injection patterns.

For the normalized resistance change approach, the electrical resistance measurements were only performed on the current injection paths corresponding to each current pattern. A 
current was sourced between two electrodes and the potential difference between both electrodes was measured. Resistance was calculated using Ohm's law. Two resistance values were collected for each electrode pair, for the adjacent and across current patterns, because the current was sourced in both directions. An average of the two values was used. Measurements were collected before and after damage initiation and normalized according to Equation 10.

$$
\frac{\Delta R}{R_{0}}=\frac{R_{\text {Damaged }}-R_{\text {Undamaged }}}{R_{\text {Undamaged }}}
$$

Table 2 shows the number of normalized resistance measurements and number of EIT voltage measurements required for each current injection pattern.

Table 2: Number of measurements for each current injection pattern

\begin{tabular}{ccc}
\hline Pattern & $\Delta \mathbf{R} / \mathbf{R}_{\mathbf{0}}$ & EIT \\
\hline \hline Adjacent & 30 & 870 \\
Across & 30 & 840 \\
Diagonal & 27 & 756 \\
\hline
\end{tabular}

\subsubsection{Ranking of Current Patterns}

Each current pattern was characterized by its ability to accurately locate the damaged region. The location of the center of damage was determined using the center of mass equations. The coordinates denoting the center of the damaged region are defined as the sum of the negative conductivity change of an individual mesh element multiplied by the element centroid and divided by the sum of all negative conductivity changes (Equation 11).

$$
x_{c m}=\frac{\sum_{i=1}^{N} m_{i} x_{i}}{M}, y_{c m}=\frac{\sum_{i=1}^{N} m_{i} y_{i}}{M}
$$




\section{Results and Discussion}

\subsection{Localized Damage Assessment}

Figures $4 \mathrm{a}, \mathrm{b}$ and 5a,b show the normalized resistance change measurements of the across current pattern for the centered and offset hole, respectively. The corresponding laminates are shown in Figures $4 \mathrm{c}$ and $5 \mathrm{c}$. The bar graphs are oriented to show the normalized resistance change for the horizontally and vertically aligned electrode pairs. Electrode 8 was set to ground, so the normalized resistance change is zero for pairs that include the $8^{\text {th }}$ electrode.

The sensitivity of the electrical network to site-specific damage is evident in both figures. In Figures 4a,b, the normalized resistance change in both directions are largest for electrode pairs surrounding the center hole, and in Figures 5a,b, the normalized resistance change are largest for electrodes aligned with the offset hole. For both damage locations the magnitude of the normalized resistance change is larger when current is sourced in the fiber direction, which is the direction of higher conductivity. This is consistent with $[35,36]$, which indicates current spreading is more significant when current is injected in the transverse direction. Large current spreading results in a more uniform potential distribution.

Figure 6 shows EIT reconstructions for each current injection pattern. The location of the drilled hole is depicted on the graph as a black, dotted-line circle. The calculated center of damage from the normalized resistance measurements is indicated by a small blue circle. The calculated center of damage of the EIT reconstruction is indicated by a red cross.

The scale showing the magnitude of the conductivity change varies by an order of magnitude depending on whether the damage occurs near the center or the boundary. The theoretical conductivity change should be -100 because the conductivity of the hole is 0 . The difference can be attributed to the linear approximation imposed by the algorithm used for the 
inverse problem. The conductivity change is outside of the conductivity range in which a quantitative solution can be obtained. Therefore, the EIT algorithm provides a qualitative assessment of damage location.

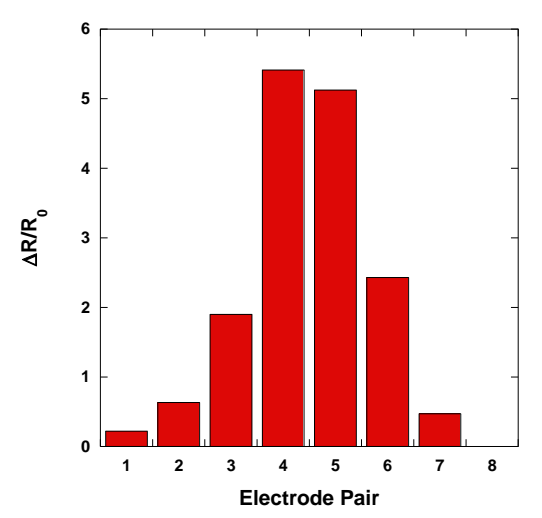

(a)

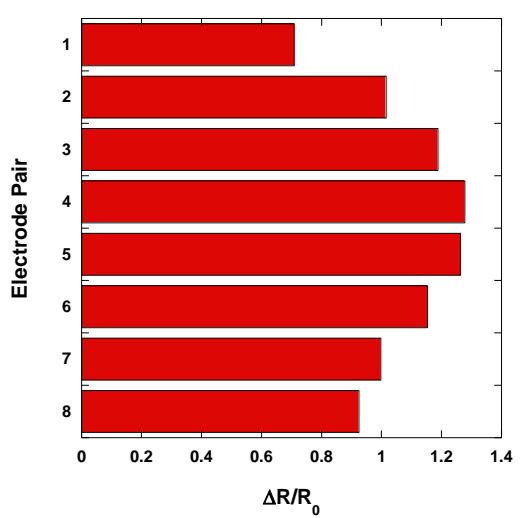

(b)

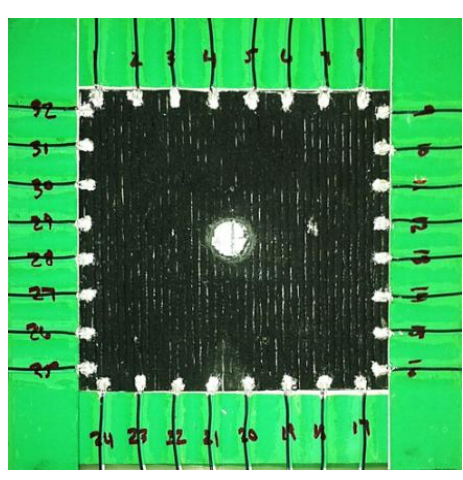

(c)

Figure 4: Normalized resistance measurements corresponding to electrodes oriented (a) horizontally and (b) vertically. (c) Photograph after a center hole was drilled.

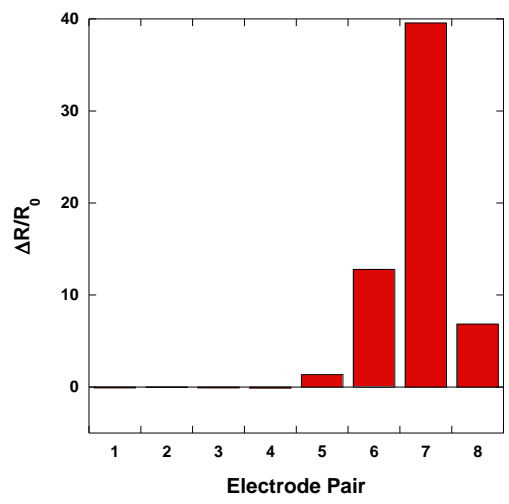

(a)

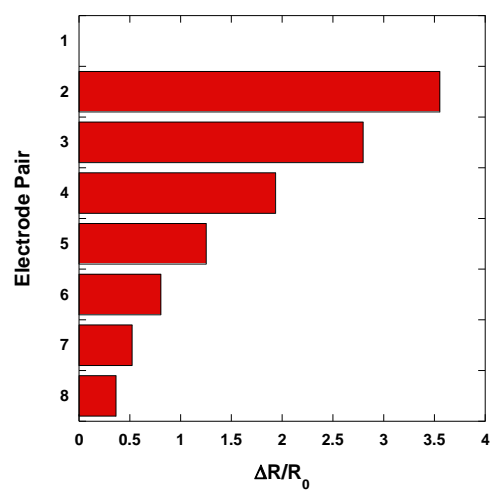

(b)

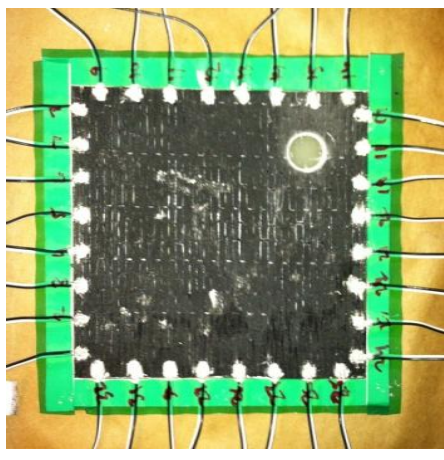

(c)

Figure 5: Normalized resistance measurements corresponding to electrodes oriented (a) horizontally and (b) vertically. (c) Photograph after an offset hole was drilled. 
Center Damage

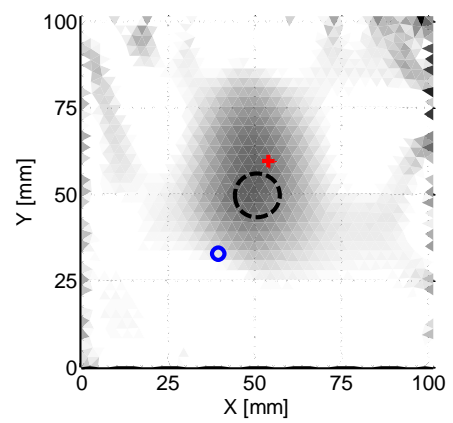

(a)

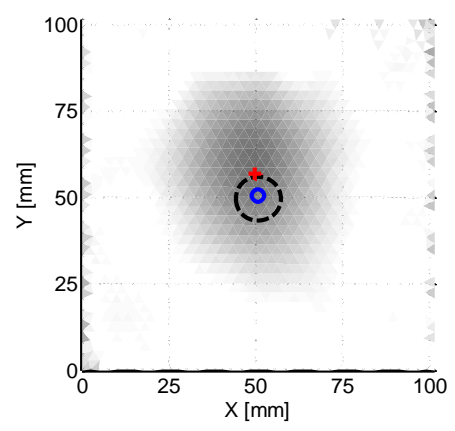

(b)

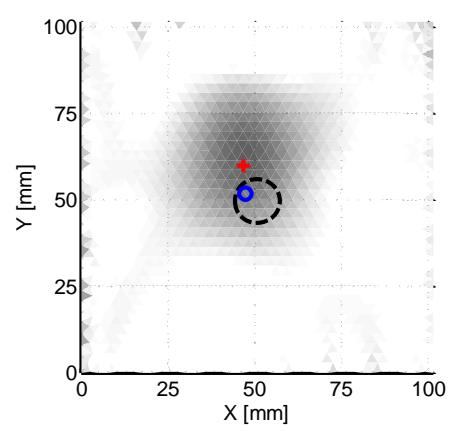

(c)

Electrical Conductivity Change [\%]

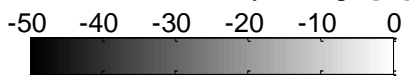

Offset Damage

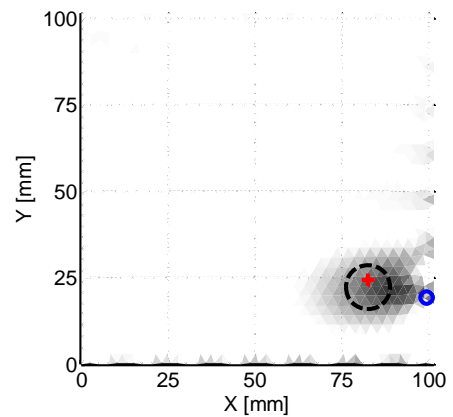

(d)

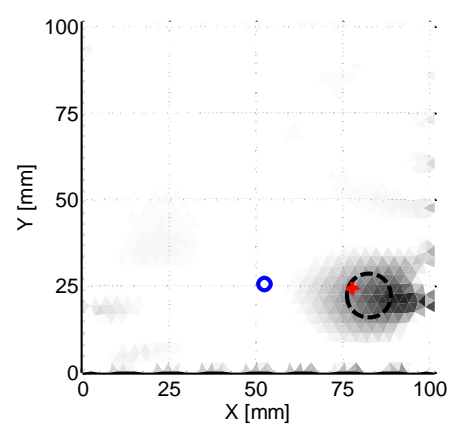

(e)

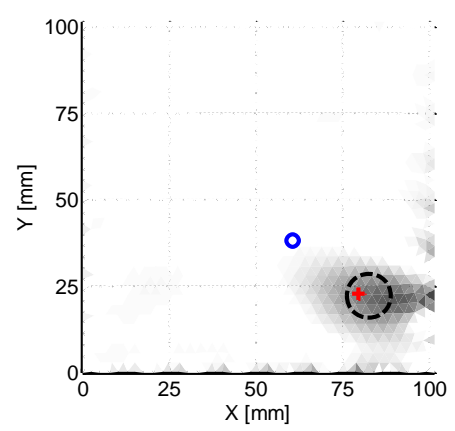

(f)

Electrical Conductivity Change [\%]

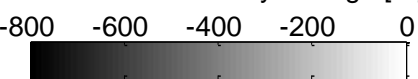

Figure 6: EIT reconstructions of a single-ply E-Glass/EPON 862/CNT composites with a hole drilled in the (a ) - (c) center and (d) - (f) offset locations. The hole location is represented by a black circle. The resistance damage center is marked by a blue ' 0 '. The EIT damage center is marked by a red ' + '. The color bars correspond to the plots in their respective columns. 
Table 2 shows the distance from the calculated center of damage for the normalized resistance measurements and EIT reconstruction to the actual center of damage. In each case, the EIT reconstruction more accurately locates the damage center but requires considerably more measurements and computational time, making real-time monitoring difficult. The normalized resistance provided a reasonable approximation of the spatial location of damage and holds potential for real-time monitoring. For EIT, each current pattern provided an accurate approximation of the damage center, and the anisotropy ratio of the laminate was low enough to avoid potential differences of zero at the boundary. From Table 2, the adjacent pattern is more sensitive to defects occurring near the electrodes while the across and diagonal patterns show greater sensitivity towards the center. Of the three current patterns, the spatial accuracy of the across pattern was the most consistent. Therefore, the across current injection pattern was used for the remainder of the study.

Table 2: Measured distance from calculated compared to the actual damage center.

\begin{tabular}{ccccc}
\hline & \multicolumn{2}{c}{ Distance (Center Hole) [mm] } & \multicolumn{2}{c}{ Distance (Offset Hole) [mm] } \\
\hline \hline Current Pattern & $\Delta \mathbf{R} / \mathbf{R}_{\mathbf{0}}$ & EIT & $\Delta \mathbf{R}_{\mathbf{R}} \mathbf{R}_{\mathbf{0}}$ & $\mathbf{E I T}$ \\
Adjacent & 20.2 & 10.5 & 17.1 & 2.20 \\
Across & 1.02 & 7.25 & 30.2 & 5.14 \\
Diagonal & 4.15 & 11.0 & 27.2 & 8.31 \\
\hline
\end{tabular}

\subsection{Impact Study}

Figure 7 shows the normalized resistance change in the vertical and horizontal directions along with an ultrasonic C-scan of the $[0 / 90]_{\mathrm{s}}$ laminate following a $20 \mathrm{~J}$ impact. The third 
electrode pair in Figure $7 \mathrm{~b}$ contained the ground electrode, and the resistance change is zero. The resistance change magnitudes in Figures $7 \mathrm{~b}$ and $\mathrm{c}$ are relatively similar. This is expected as the transverse and longitudinal conductivity is similar due to the cross-ply layup. The damage location inferred from the normalized resistance plots is consistent with the C-scanned image. Figure 8 shows the reconstructed conductivity of the panel following impacts of $10 \mathrm{~J}$ and $20 \mathrm{~J}$. The damage center predicted by the EIT measurements is closest to the actual impact site in Figure 8a. A benefit to using EIT for damage location is that the technique is highly sensitive in detecting small changes in conductivity and, therefore, accurately locates small-scale matrix damage. The subsequent impact at $20 \mathrm{~J}$ results in much larger-scale damage, significantly affecting the resistance measurements. The damage center predicted by the normalized resistance change is actually slightly more accurate than EIT.

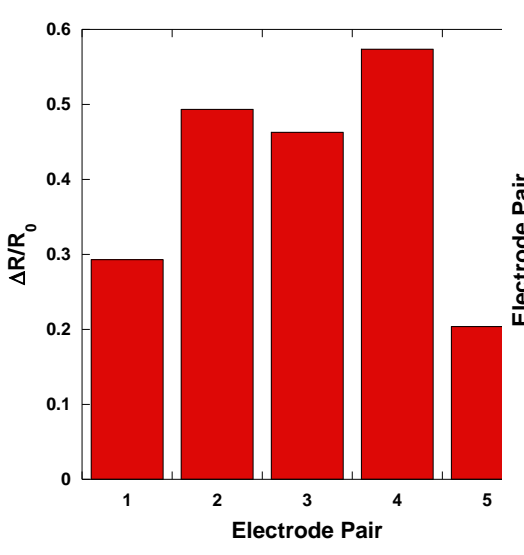

(a)

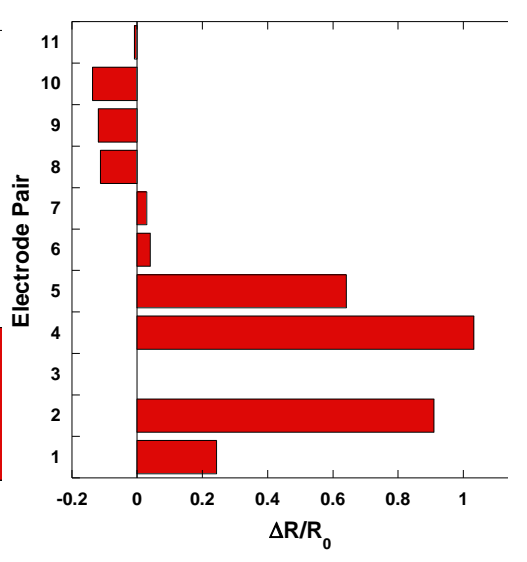

(b)

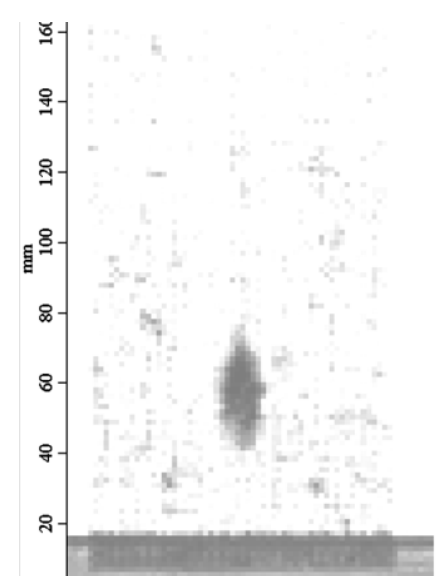

(c)

Figure 7: Normalized resistance measurements corresponding to electrodes oriented (a) horizontally and (b) vertically. (c) The C-scan of the panel following an impact of $20 \mathrm{~J}$. 


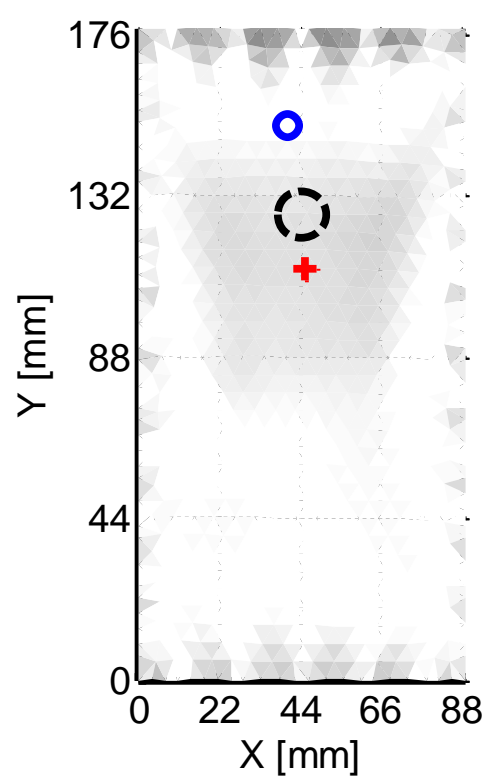

(a)

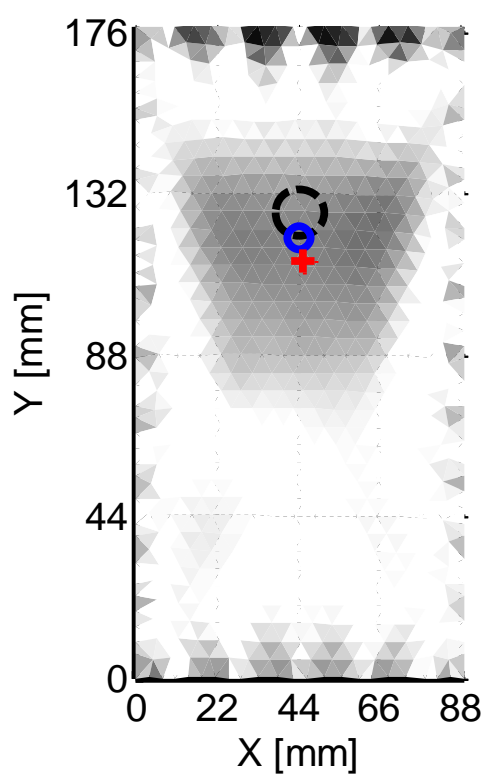

(b)

Electrical Conductivity Change [\%]

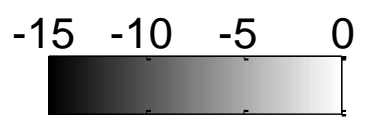

Figure 8: EIT reconstructions of a single-ply E-Glass/EPON 862/CNT composite following an impact of (a) $10 \mathrm{~J}$ and (b) $20 \mathrm{~J}$. The hole location is represented by a black circle. The resistance damage center is marked by a blue ' 0 '. The EIT damage center is marked by a red ' + '. The color bars correspond to the plots in their respective columns.

\subsection{Tensile Delamination Study}

Figure 9 shows the C-scan showing the transverse cut. The ply discontinuity was approximately $100 \mathrm{~mm}$ from the left edge of the laminate, between electrodes 9 and 10, and 27 and 28. Ply delamination will initiate at the ply discontinuity under tensile load. Figure 10 shows the normalized resistance response following the $44.5 \mathrm{kN}$ loading cycle. This initiation of delamination was detected using acoustic emission. The largest resistance change is observed near electrodes 9 and 28, which are next to the ply discontinuity. The resistance change is zero for the electrode 8-29 pair due to electrode 8 being used as the ground. Damage is not only located near the ply drop. Resistance measurements show noticeable changes near electrode pairs 
6-31 and 16-21. It is likely that some localized damage occurs in this area due to the end effects as well as transverse splitting of the plies under tension.

Figure 11 shows the EIT reconstruction for the across current pattern. The location of the ply discontinuity is indicated by a solid line. The location of significant ply delamination was approximated for both the resistance and EIT measurements by calculating the x-coordinate for the 'center of damage'. The location of delamination from the resistance measurements is shown by a dotted blue line, and the location based from the EIT reconstruction is indicated by a dotted red line. The normalized resistance measurements and EIT reconstruction show reasonable agreement for indicating the region of most significant damage, but the distributed damage in the tensile specimen, clearly shown by the large transverse resistance change, makes damage localization more difficult.

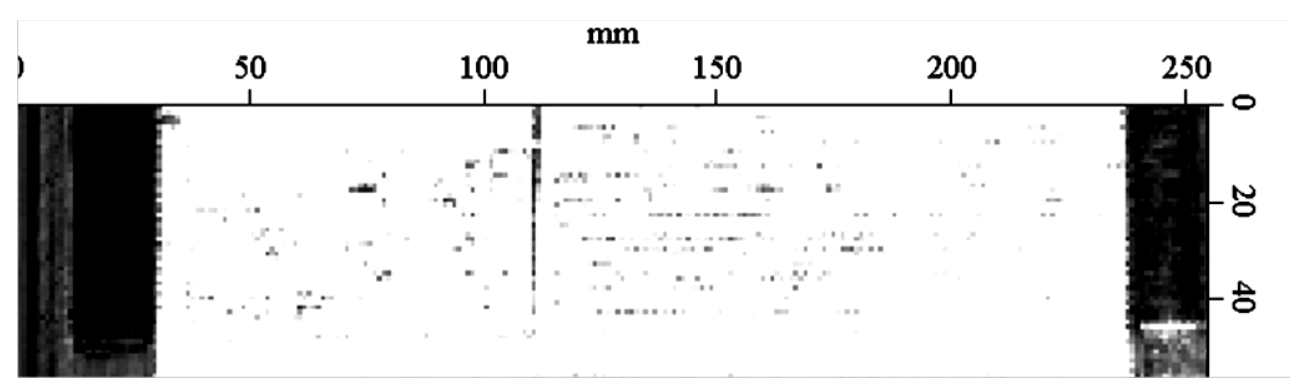

Figure 9: C-scan of $[0]_{5}$ E-Glass/EPON 862/CNT panel with the center ply cut in order to initiate delamination. 


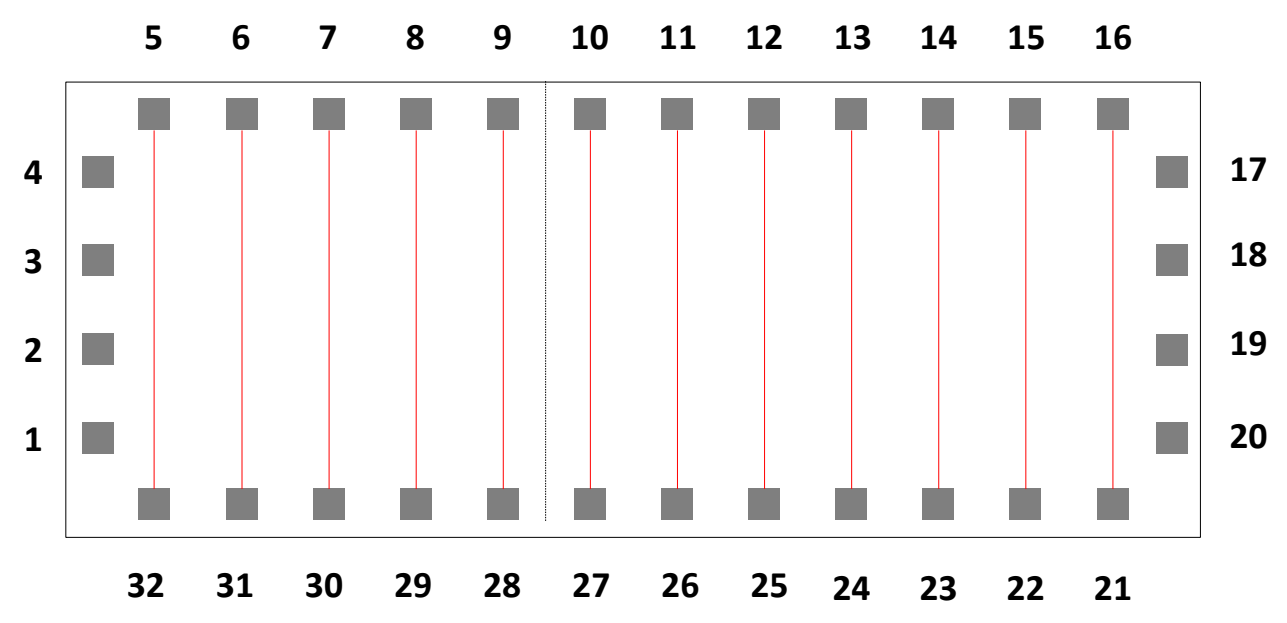

(a)

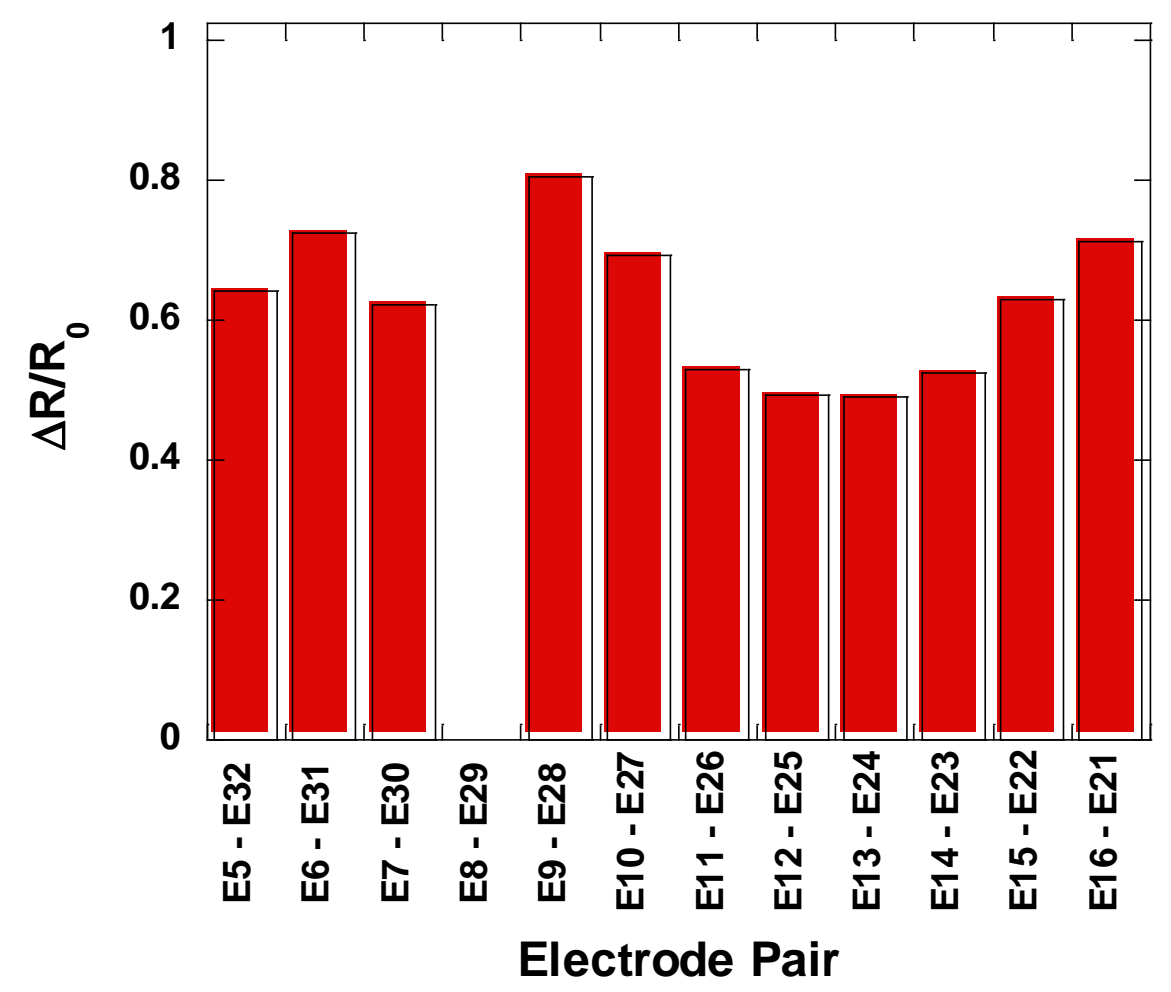

(b)

Figure 10: (a) Illustration of panel with current injection lines for normalized resistance measurements and the ply discontinuity indicated by a dashed line. (b) Normalized resistance measurements 


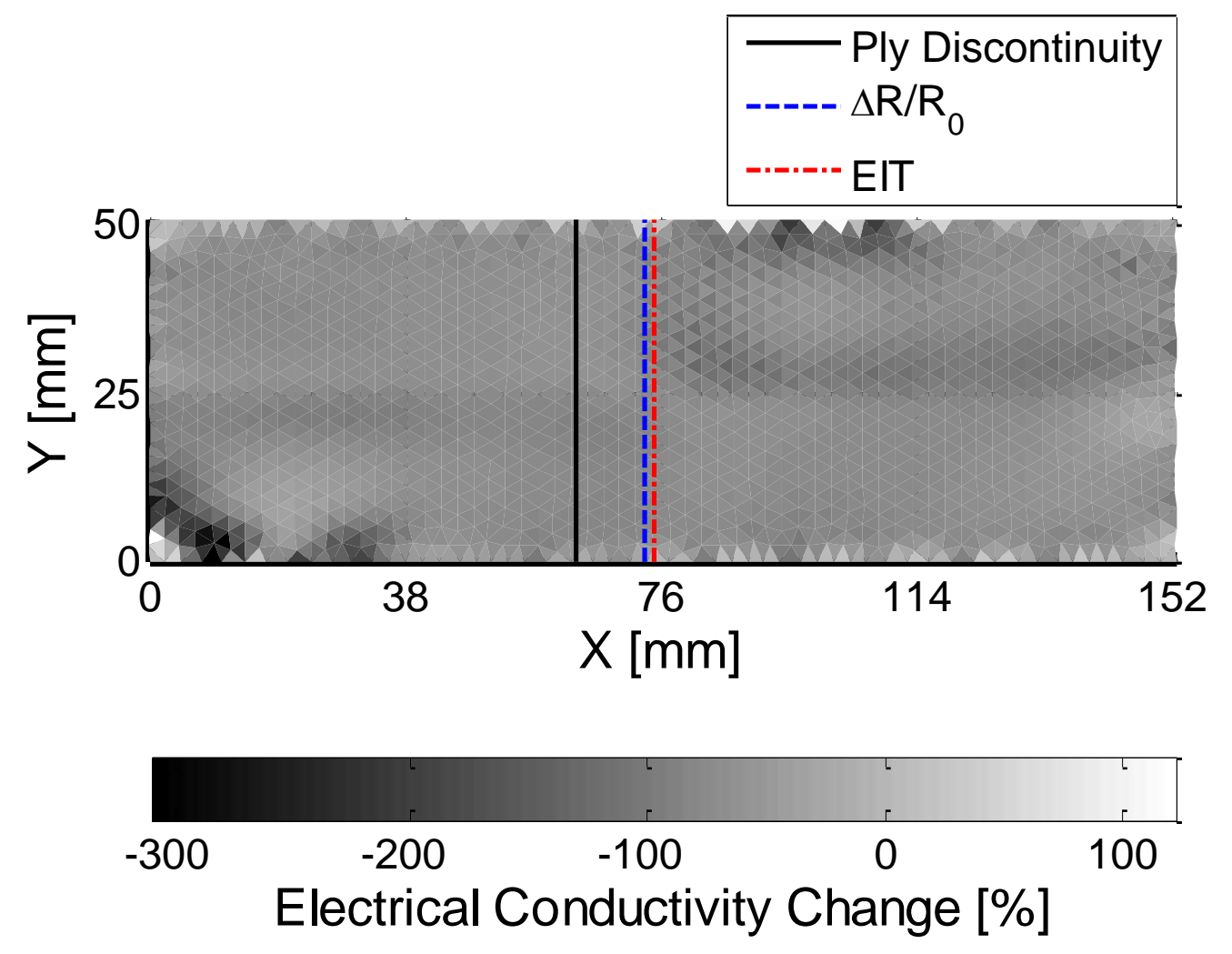

Figure 11: EIT reconstructions of the $[0]_{5}$ E-Glass/EPON 862/CNT tensile bar using the across current injection pattern.

\section{Conclusions}

Two-dimensional resistance measurements and electrical impedance tomography were demonstrated as potential SHM techniques for detecting and locating damaged regions in orthotropic fiber-reinforced composites. Resistance measurements from an electrode network provided a quick initial assessment of the damaged state. However, EIT was demonstrated to more accurately determine the location of damage. Also, the spatial resolution and accuracy of the EIT reconstruction is dependent on the type of current pattern used. Sourcing current between adjacent electrodes along the boundary resulted in higher sensitivity to defects near the edge. Current sourced across the entire length of the panel, as in the across and diagonal patterns, resulted in greater sensitivity to defects near the center. 


\section{Acknowledgements}

This work is funded by the Air Force Research Laboratory (AFRL) Award No. FA9550-11C-0003 (Phase II STTR with Acellent Technologies, Dr. David Stargel, Program Manager) and the National Science Foundation (Award \#1234830), Kishor Mehta, Program Director of HSME.

\section{References}

[1] P. K. Mallick, Fiber-Reinforced Composites: Materials, Manufacturing, and Design, Third Edition, vol. 19. CRC Press, 2007, p. 640.

[2] C. Soutis, "Fibre reinforced composites in aircraft construction," Prog. Aerosp. Sci., vol. 41, no. 2, pp. 143-151, Feb. 2005.

[3] A. R. Bunsell and J. Renard, Fundamentals of Fibre Reinforced Composite Materials. CRC Press, 2005, p. 225.

[4] M. Enckell, B. Glisic, F. Myrvoll, and B. Bergstrand, "Evaluation of a large-scale bridge strain, temperature and crack monitoring with distributed fibre optic sensors," J. Civ. Struct. Heal. Monit., vol. 1, no. 1-2, pp. 37-46, Mar. 2011.

[5] Y. . Rao, "Recent progress in applications of in-fibre Bragg grating sensors," Opt. Lasers Eng., vol. 31, no. 4, pp. 297-324, Apr. 1999.

[6] H. Ling, K. Lau, L. Cheng, and W. Jin, "Viability of using an embedded FBG sensor in a composite structure for dynamic strain measurement," Measurement, vol. 39, no. 4, pp. 328-334, May 2006.

[7] A. RAGHAVAN and C. E. S. CESNIK, "Review of guided-wave structural health monitoring," Shock Vib. Dig., vol. 39, no. 2, pp. 91-114.

[8] K. Schulte and C. Baron, "Load and failure analyses of CFRP laminates by means of electrical resistivity measurements," Compos. Sci. Technol., vol. 36, no. 1, pp. 63-76, Jan. 1989.

[9] E. T. Thostenson and T.-W. Chou, "Carbon Nanotube Networks: Sensing of Distributed Strain and Damage for Life Prediction and Self Healing," Adv. Mater., vol. 18, no. 21, pp. 2837-2841, 2006.

[10] L. Gao, E. T. Thostenson, Z. Zhang, and T.-W. Chou, "Sensing of Damage Mechanisms in Fiber-Reinforced Composites under Cyclic Loading using Carbon Nanotubes," Adv. Funct. Mater., vol. 19, no. 1, pp. 123-130, Jan. 2009. 
[11] A. Todoroki, Y. Tanaka, and Y. Shimamura, "Delamination monitoring of graphite/epoxy laminated composite plate of electric resistance change method," Compos. Sci. Technol., vol. 62, no. 9, pp. 1151-1160, Jul. 2002.

[12] A. Todoroki, "High performance estimations of delamination of graphite/epoxy laminates with electric resistance change method," Compos. Sci. Technol., vol. 63, no. 13, pp. 19111920, Oct. 2003.

[13] N. Angelidis, N. Khemiri, and P. E. Irving, "Experimental and finite element study of the electrical potential technique for damage detection in CFRP laminates," Smart Mater. Struct., vol. 14, pp. 147-154, 2005.

[14] R. Schueler, S. P. Joshi, and K. Schulte, "Damage detection in CFRP by electrical conductivity mapping," Compos. Sci. Technol., vol. 61, no. 6, pp. 921-930, May 2001.

[15] T.-C. Hou, K. J. Loh, and J. P. Lynch, "Spatial conductivity mapping of carbon nanotube composite thin films by electrical impedance tomography for sensing applications," Nanotechnology, vol. 18, no. 31, p. 315501, Aug. 2007.

[16] K. J. Loh, T.-C. Hou, J. P. Lynch, and N. A. Kotov, "Carbon Nanotube Sensing Skins for Spatial Strain and Impact Damage Identification,” J. Nondestruct. Eval., vol. 28, no. 1, pp. 9-25, Mar. 2009.

[17] A. Baltopoulos, N. Polydorides, L. Pambaguian, A. Vavouliotis, and V. Kostopoulos, "Damage identification in carbon fiber reinforced polymer plates using electrical resistance tomography mapping," J. Compos. Mater., vol. 47, no. 26, pp. 3285-3301, Oct. 2012.

[18] B. R. Loyola, T. M. Briggs, L. Arronche, K. J. Loh, V. La Saponara, G. O’Bryan, and J. L. Skinner, "Detection of spatially distributed damage in fiber-reinforced polymer composites," Struct. Heal. Monit., vol. 12, no. 3, pp. 225-239, Apr. 2013.

[19] “SIZICYL XC R2G Product Data,” 2009.

[20] L. Gao, T.-W. Chou, E. T. Thostenson, A. Godara, Z. Zhang, and L. Mezzo, "Highly conductive polymer composites based on controlled agglomeration of carbon nanotubes," Carbon N. Y., vol. 48, no. 9, pp. 2649-2651, Aug. 2010.

[21] G. J. Gallo and E. T. Thostenson, "Electrical characterization and modeling of carbon nanotube and carbon fiber self-sensing composites for enhancing sensing of microcracks," Mater. Today Commun., 2015.

[22] D. S. Holder, Electrical Impedance Tomography: Methods, History and Applications. IOP Publishing Ltd., 2005. 
[23] J.-F. P. J. Abascal, S. R. Arridge, D. Atkinson, R. Horesh, L. Fabrizi, M. De Lucia, L. Horesh, R. H. Bayford, and D. S. Holder, "Use of anisotropic modelling in electrical impedance tomography: description of method and preliminary assessment of utility in imaging brain function in the adult human head.," Neuroimage, vol. 43, no. 2, pp. 258-68, Nov. 2008.

[24] M. Cheney, D. Isaacson, and J. C. Newell, "Electrical Impedance Tomography," SIAM Rev., vol. 41, no. 1, pp. 85-101, Jan. 1999.

[25] M. Hallaji, A. Seppänen, and M. Pour-Ghaz, "Electrical impedance tomography-based sensing skin for quantitative imaging of damage in concrete," Smart Mater. Struct., vol. 23, no. 8, p. 085001, Aug. 2014.

[26] A. Adler and R. Guardo, "Electrical impedance tomography: regularized imaging and contrast detection.," IEEE Trans. Med. Imaging, vol. 15, no. 2, pp. 170-9, Jan. 1996.

[27] A. Adler and W. R. B. Lionheart, "Uses and abuses of EIDORS: an extensible software base for EIT.," Physiol. Meas., vol. 27, no. 5, pp. S25-42, May 2006.

[28] "Fundamentals of RF and Microwave Noise Figure Measurements." Agilent Technologies.

[29] P.-O. Persson and G. Strang, "A simple mesh generator in MATLAB," SIAM Rev., vol. 46, no. 2, pp. 329-345, 2004.

[30] N. Polydorides and W. Lionheart, "A Matlab tookit for three-dimensional electrical impedance tomography: a contribution to the Electrical Impedance and Diffuse Optical Reconstruction Software project," Meas. Sci. Technol., vol. 13, no. 12, 2002.

[31] M. Vauhkonen, "Electrical Impedance Tomography and Prior Information," Kuopio University, 1997.

[32] N. Polydorides, "Image Reconstruction Algorithms for Soft-Field Tomography," University of Manchester Institute of Science and Technology, 2002.

[33] P. Kauppinen, J. Hyttinen, and J. Malmivuo, "Sensitivity Distribution Visualizations of Impedance Tomography Measurement Strategies," Int. J. Bioelectromagn., vol. 8, no. 1, pp. 1-9, 2006.

[34] D. Zhang, L. Ye, D. Wang, Y. Tang, S. Mustapha, and Y. Chen, “Assessment of transverse impact damage in GF/EP laminates of conductive nanoparticles using electrical resistivity tomography," Compos. Part A Appl. Sci. Manuf., vol. 43, no. 9, pp. 1587-1598, Sep. 2012.

[35] D. Wang, S. Wang, D. D. L. Chung, and J. H. Chung, "Comparison of the Electrical Resistance and Potential Techniques for the Self-sensing of Damage in Carbon Fiber 
Polymer-Matrix Composites," J. Intell. Mater. Syst. Struct., vol. 17, no. 10, pp. 853-861, Oct. 2006.

[36] D. Wang and D. D. L. Chung, "Comparative evaluation of the electrical configurations for the two-dimensional electric potential method of damage monitoring in carbon fiber polymer-matrix composite," Smart Mater. Struct., vol. 15, no. 5, pp. 1332-1344, Oct. 2006. 


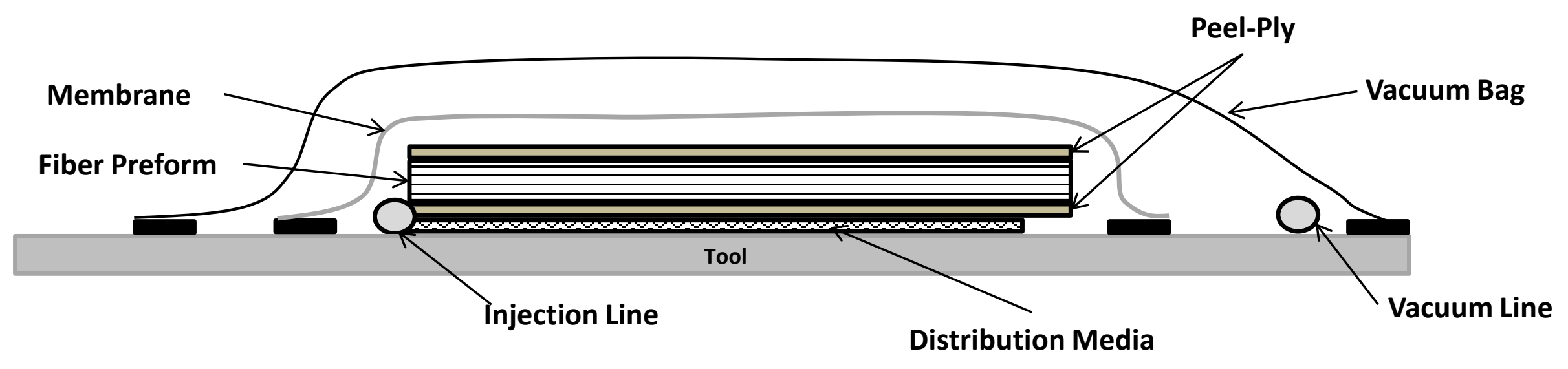

Figure 1: Illustration of the modified vacuum bag setup used for sizing fibers with nanotubes. 
Figure 2: Illustration of tape placement for electrode preparation in (a) laminates used for site-specific testing and (b) laminates used for impact testing.

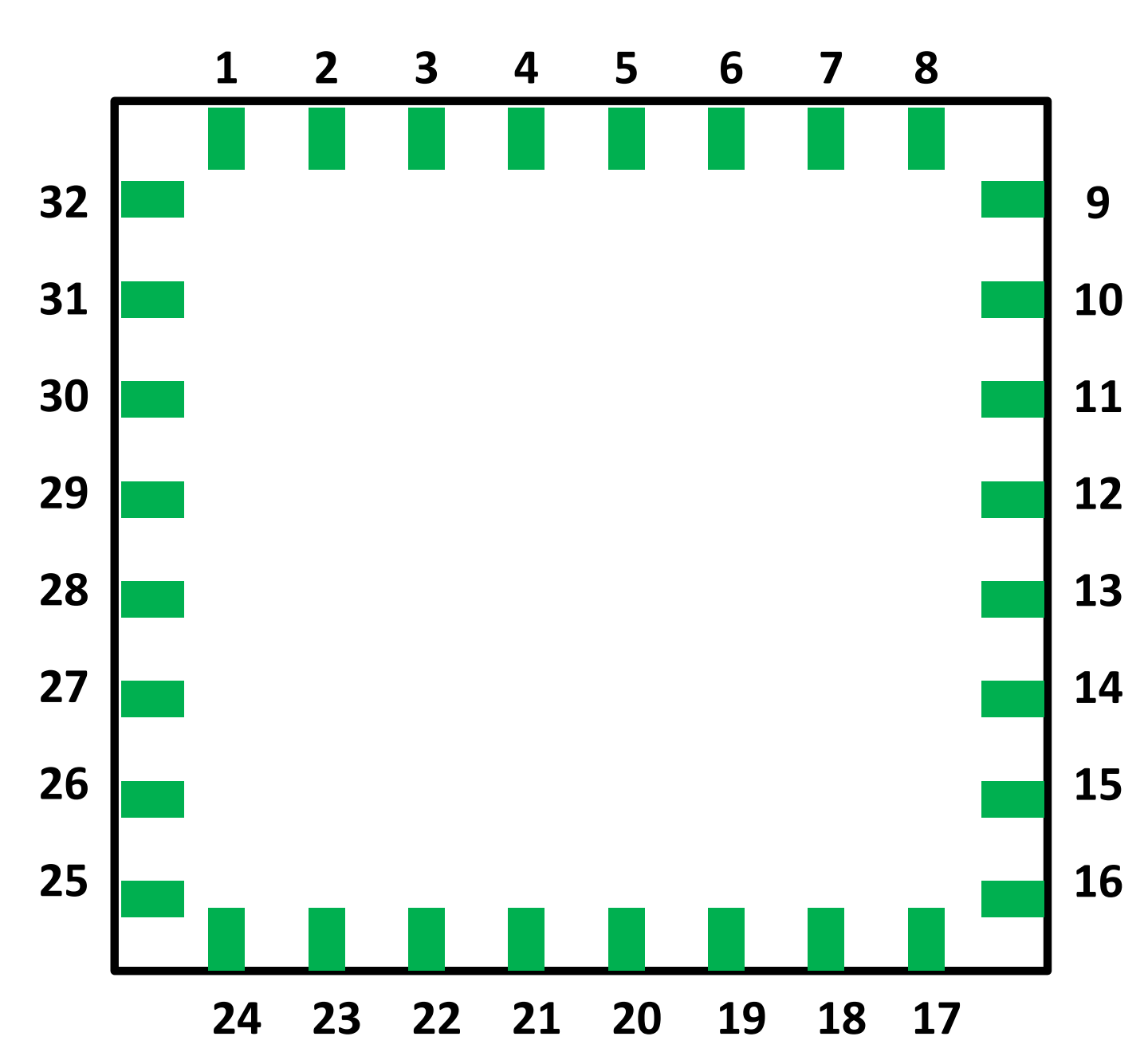

(a) 


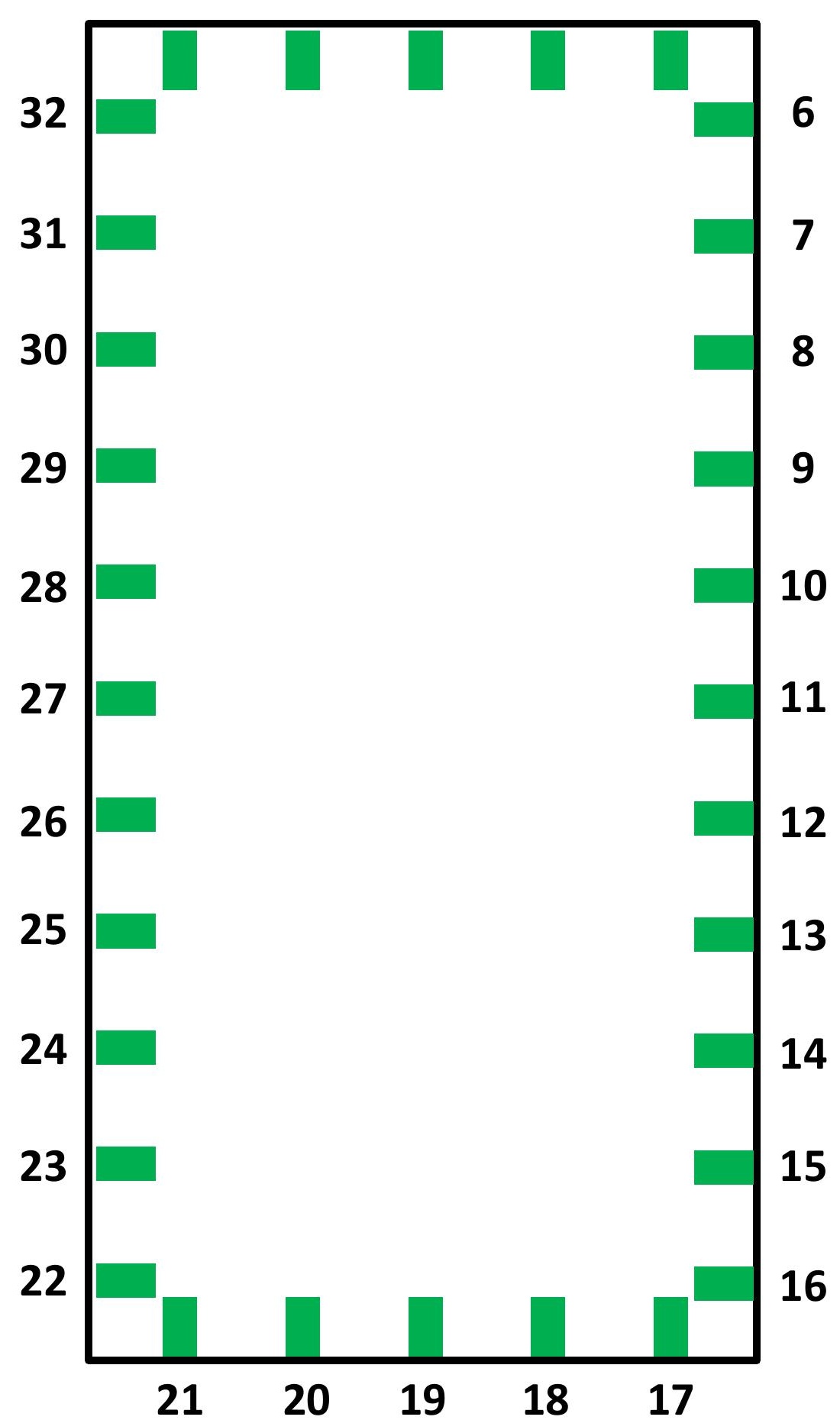


Figure 3: Illustrations of the (a) adjacent, (b) across, and (c) diagonal current injection patterns.

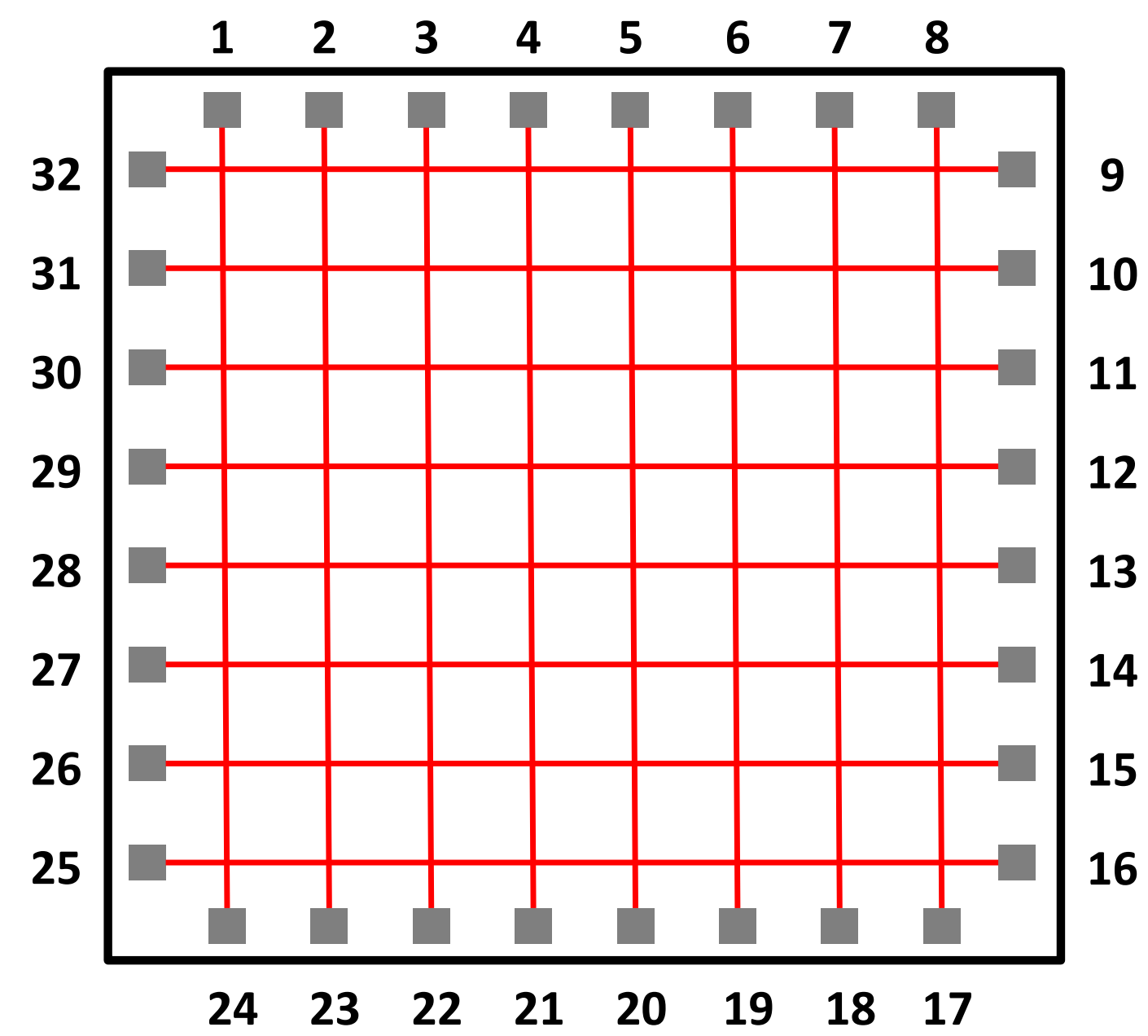

(a)

$$
\begin{array}{llllllll}
24 & 23 & 22 & 21 & 20 & 19 & 18 & 17
\end{array}
$$




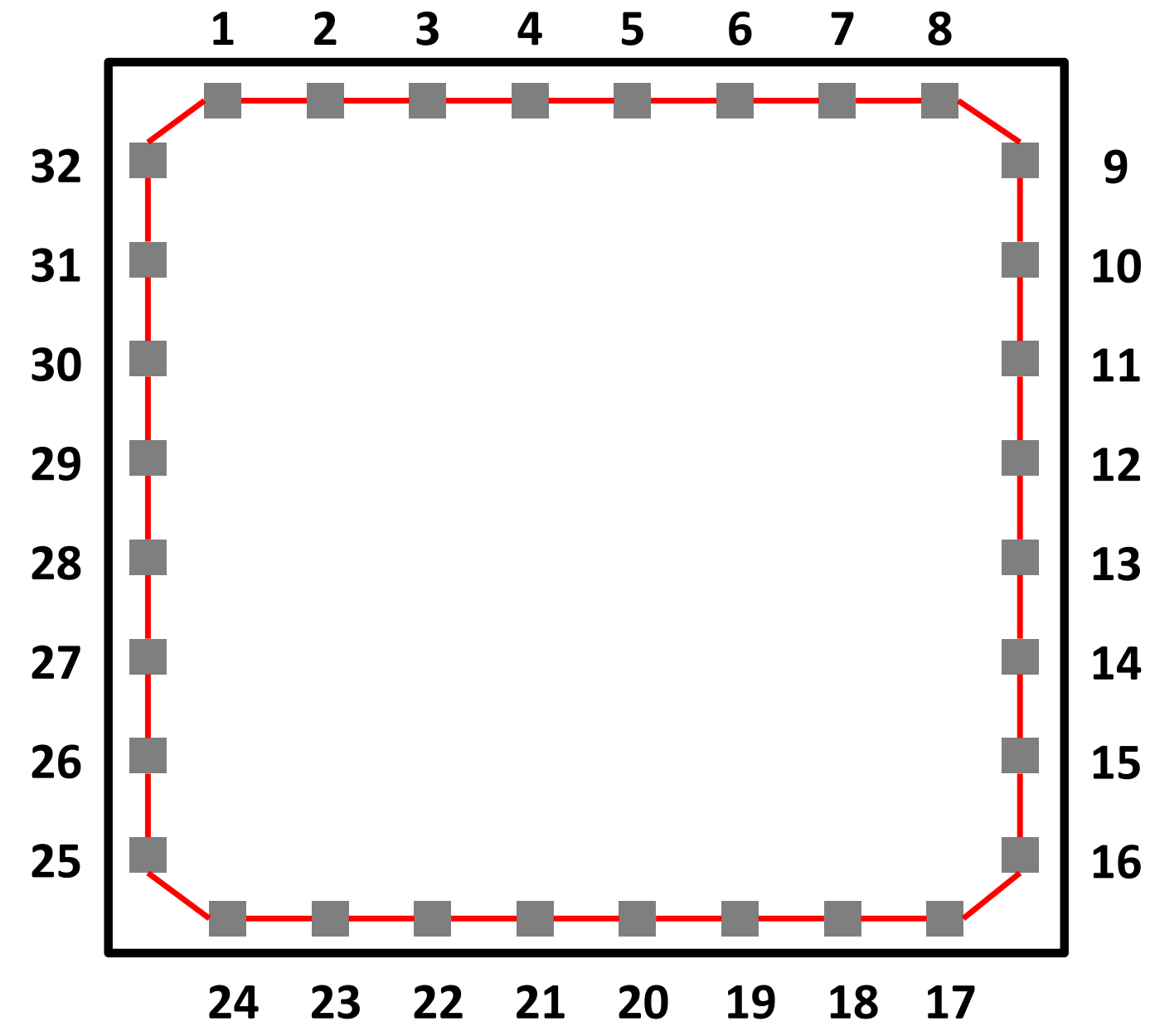

(b) 


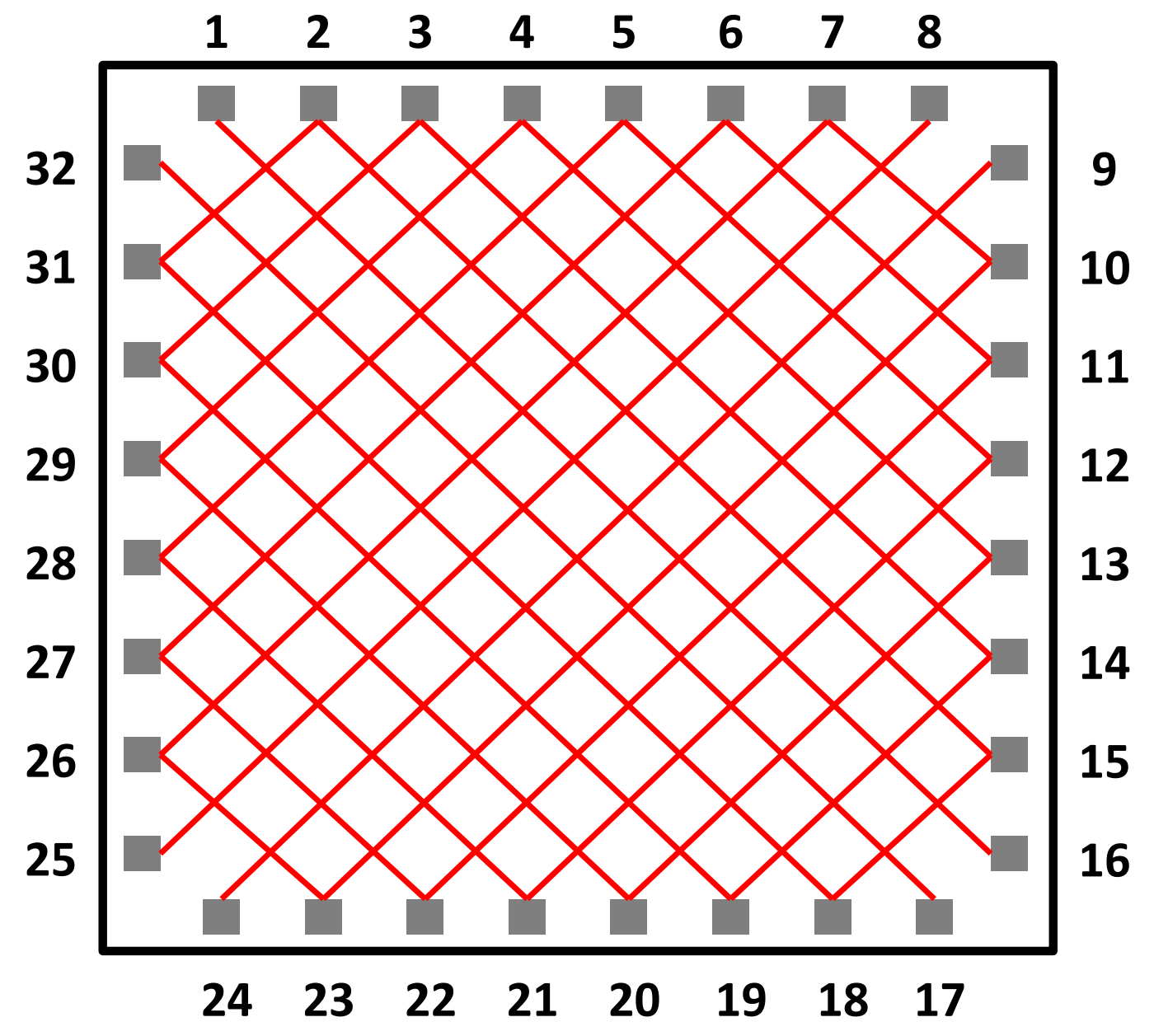

(c) 


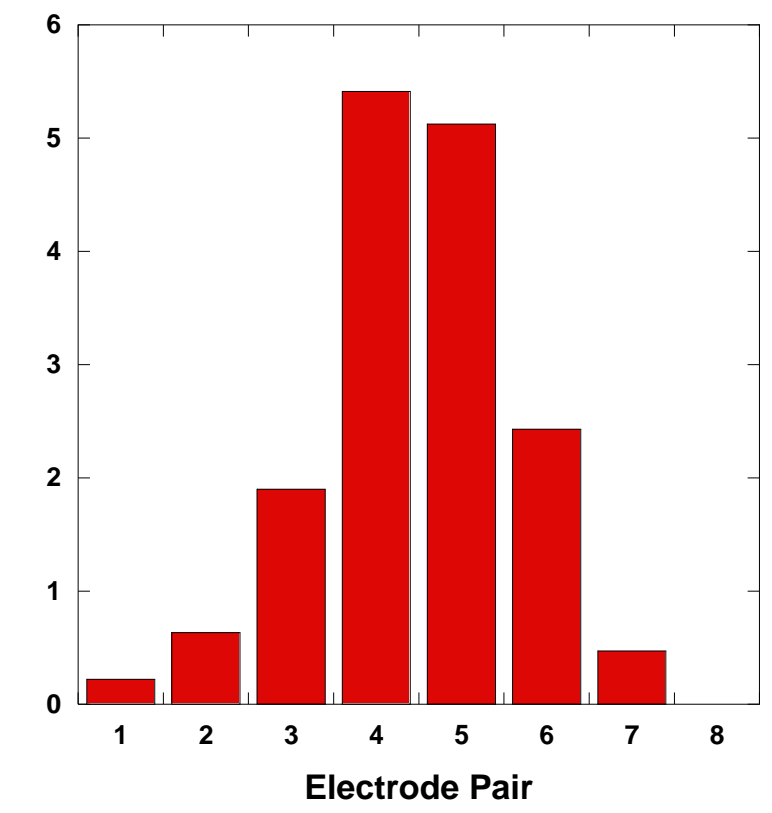

(a)

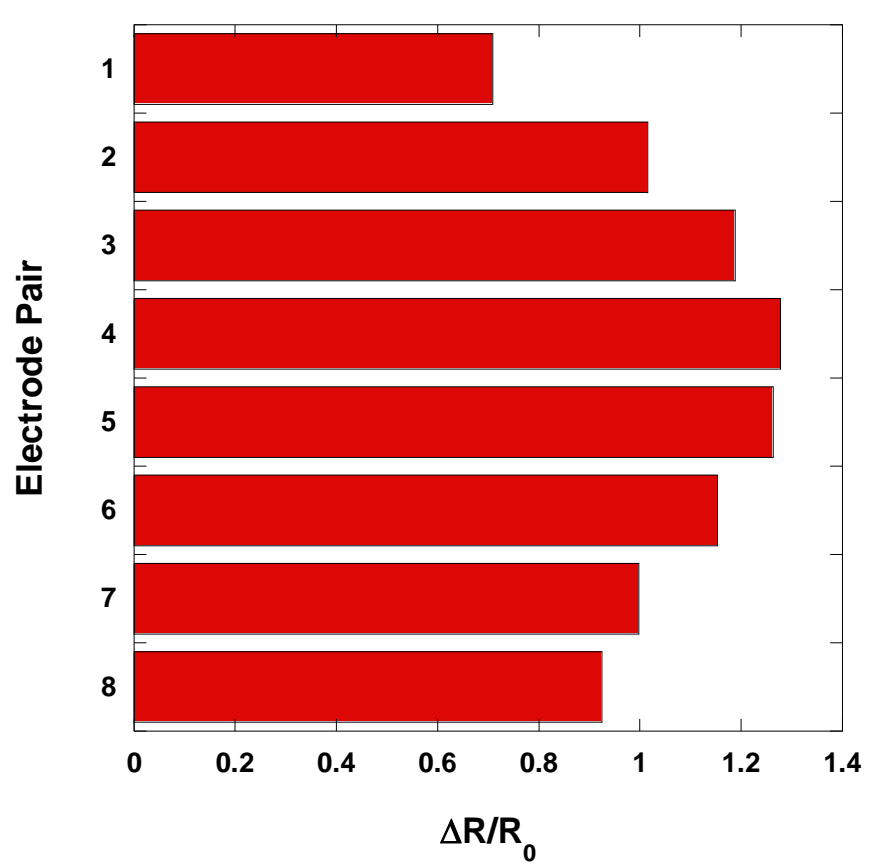

(b)

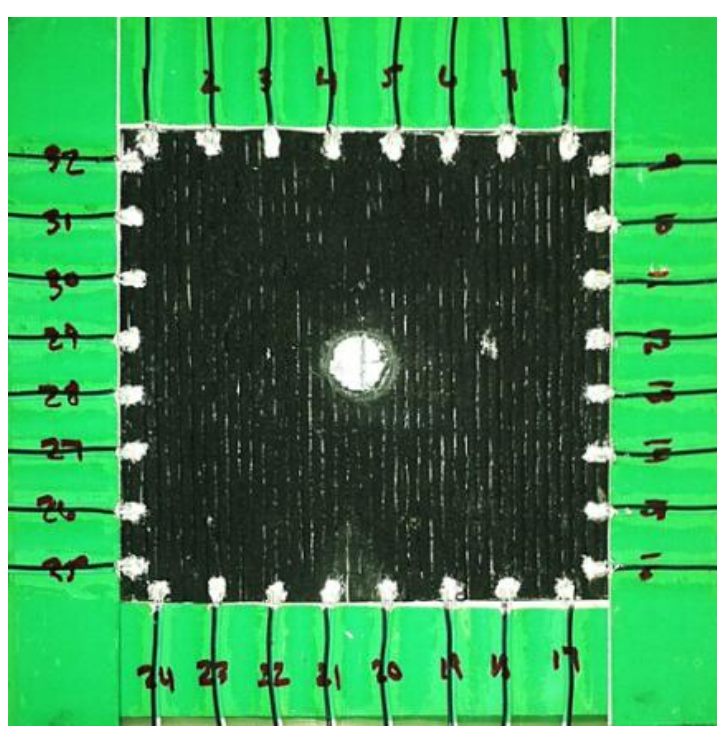

(c)

Figure 4: Normalized resistance measurements corresponding to electrodes oriented (a) horizontally and (b) vertically. (c) Photograph after a center hole was drilled. 
Figure 6: EIT reconstructions of a single-ply E-Glass/EPON 862/CNT composites with a hole drilled in the (a ) - (c) center and (d) - (f) offset locations. The hole location is represented by a black circle. The resistance damage center is marked by a blue ' $o$ '. The EIT damage center is marked by a red ' $t$ '. The color bars correspond to the plots in their respective columns.

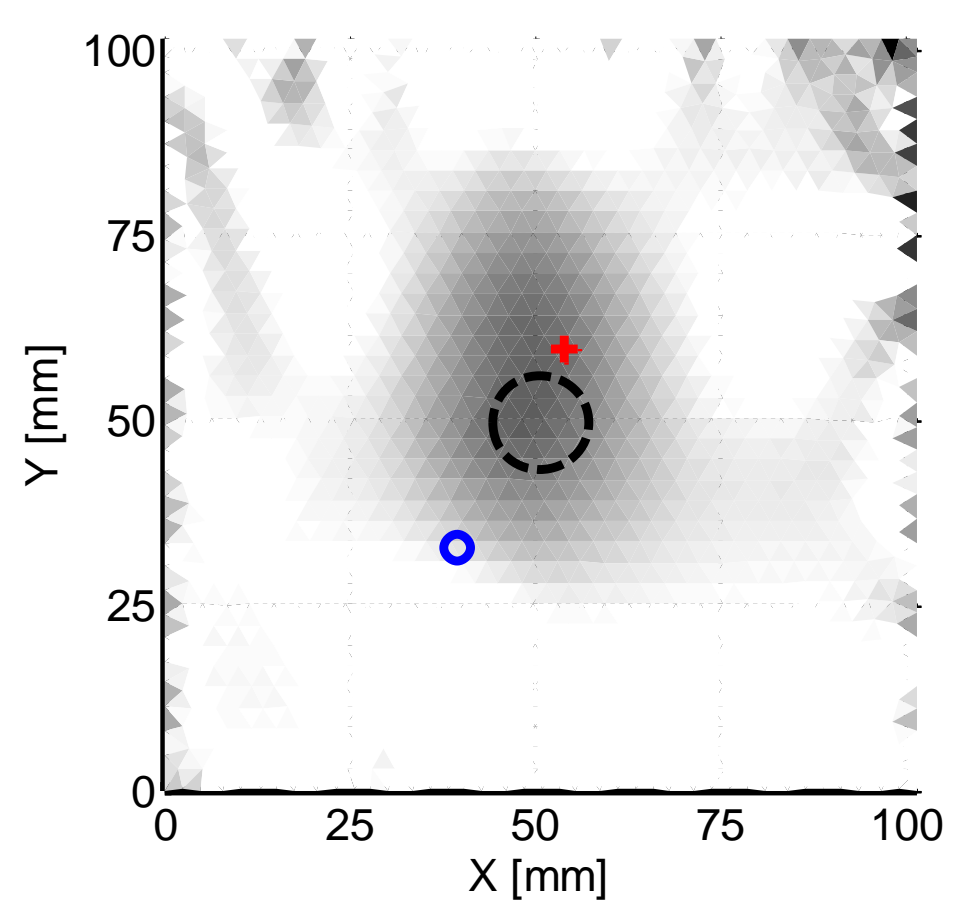

(a)

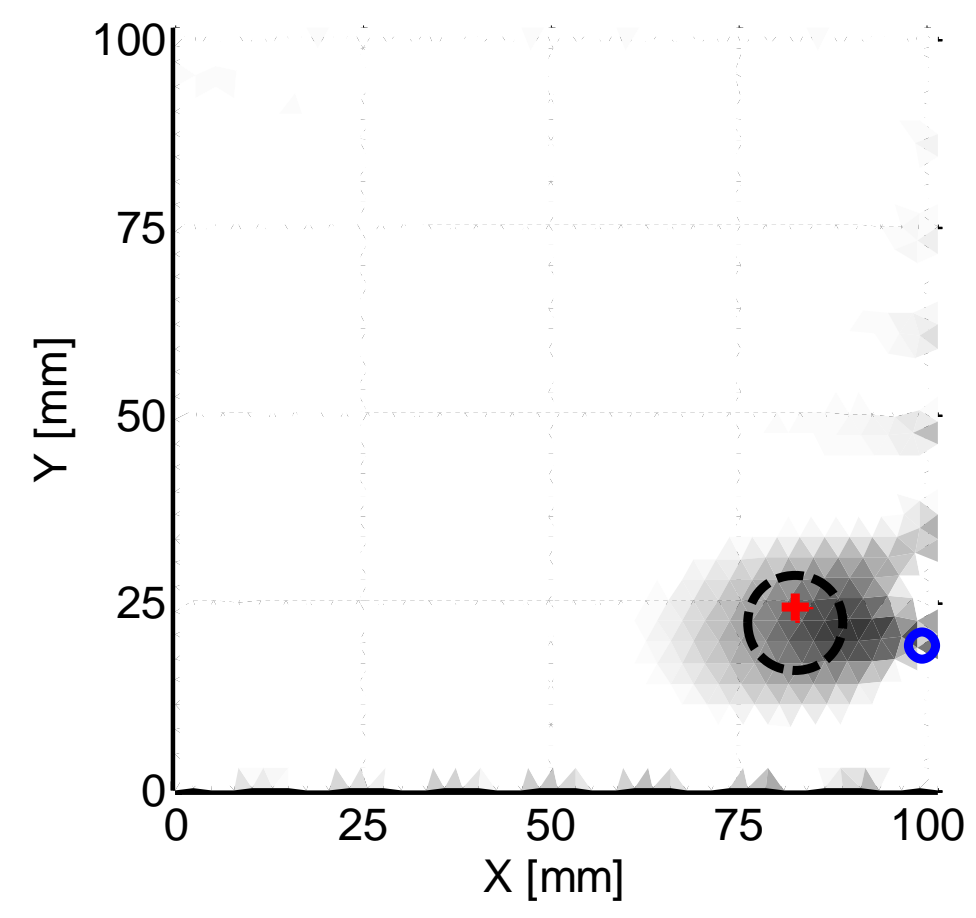

(d) 


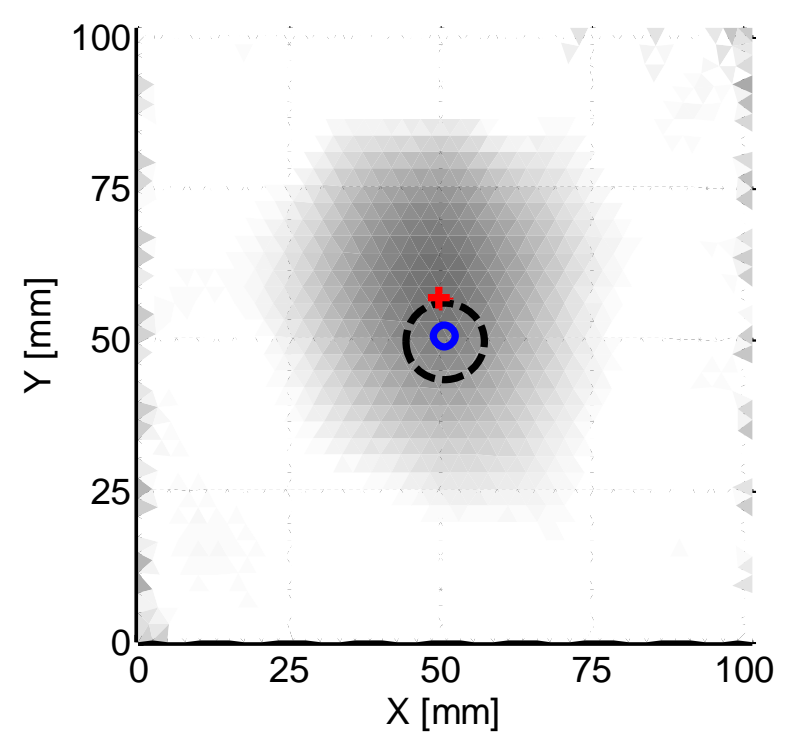

(b)

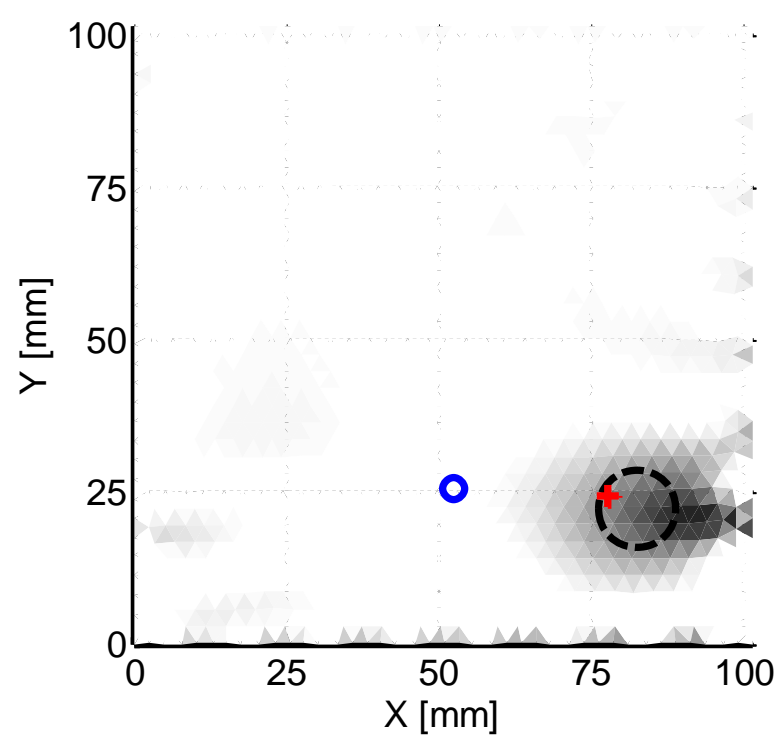

(e) 


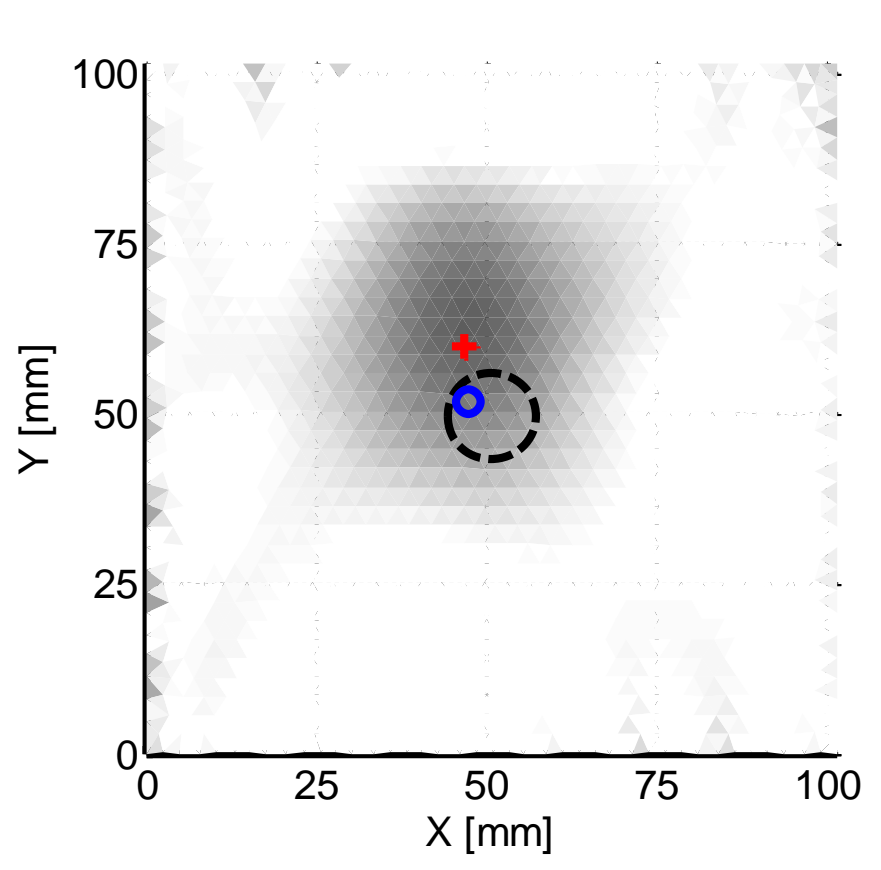

(c)

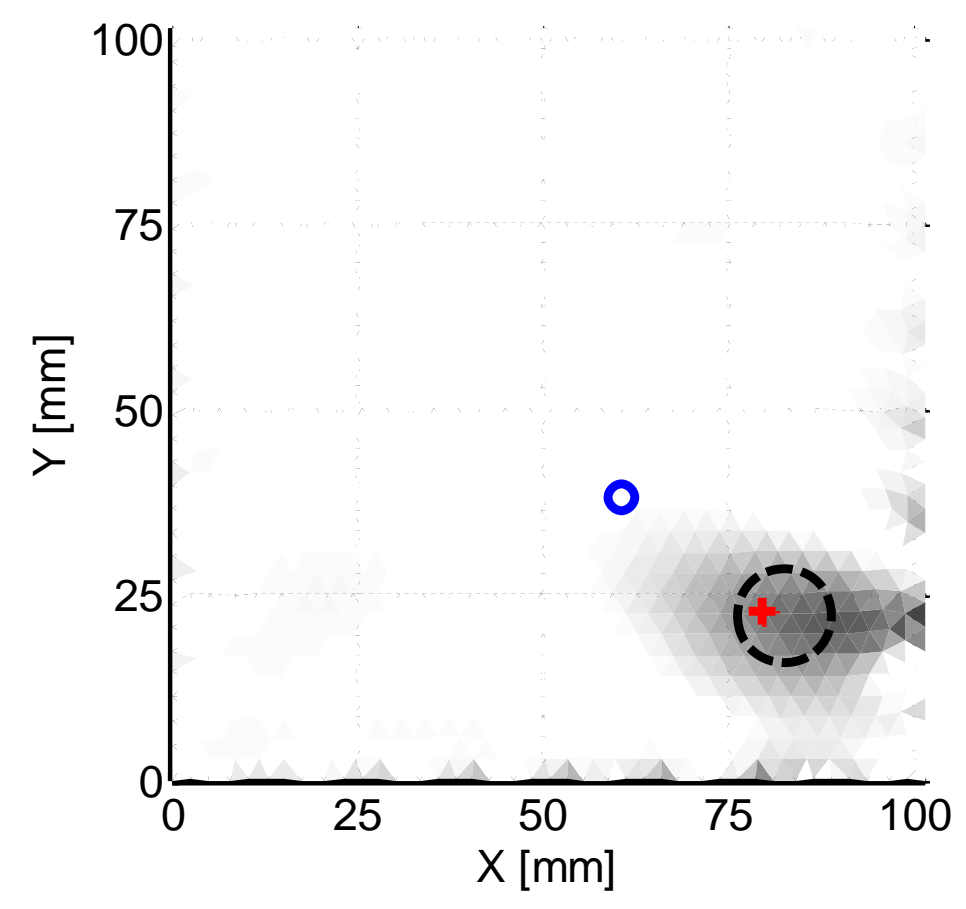

(f) 
Electrical Conductivity Change [\%] $\begin{array}{llllll}-50 & -40 & -30 & -20 & -10 & 0\end{array}$
Electrical Conductivity Change [\%] $\begin{array}{lllll}-800 & -600 & -400 & -200 & 0\end{array}$ 
Figure 7: Normalized resistance measurements corresponding to electrodes oriented (a) horizontally and (b) vertically. (c) The C-scan of the panel following an impact of $20 \mathrm{~J}$.

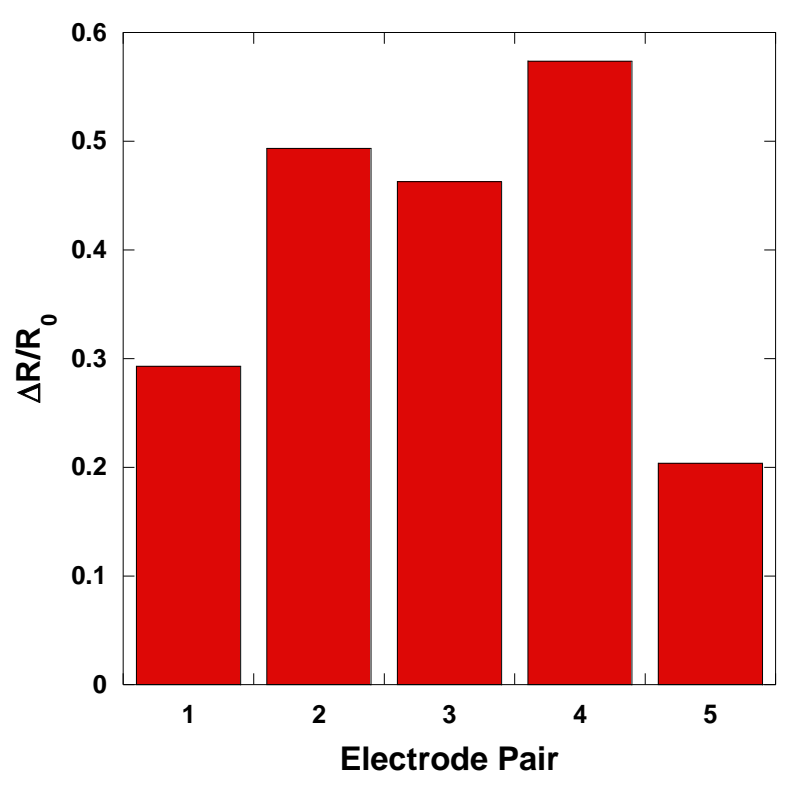

(a)

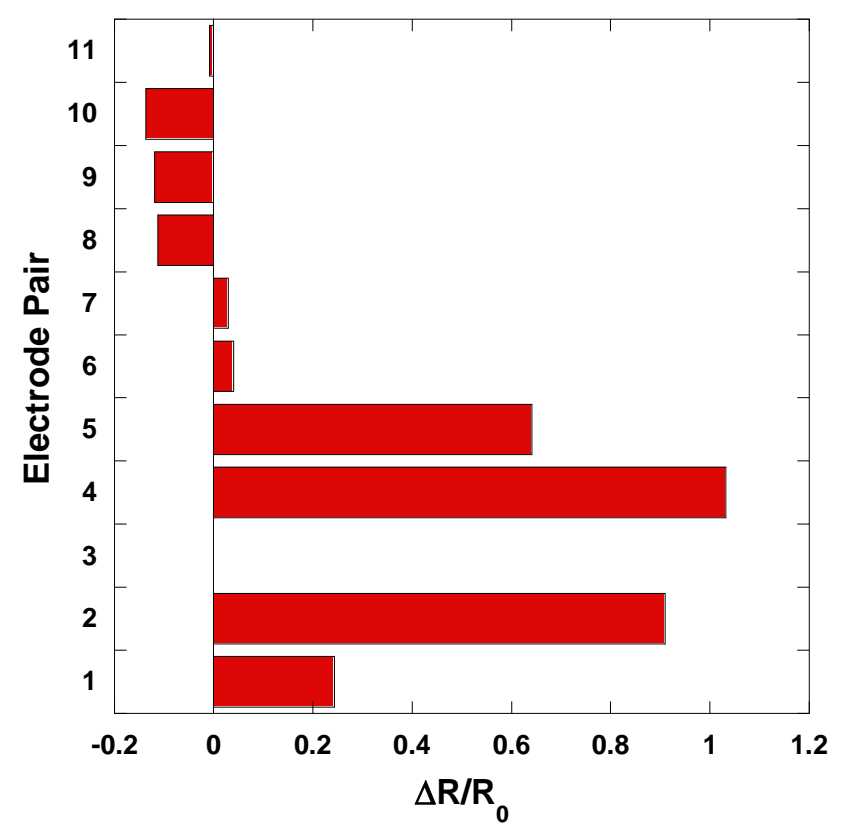

(b)

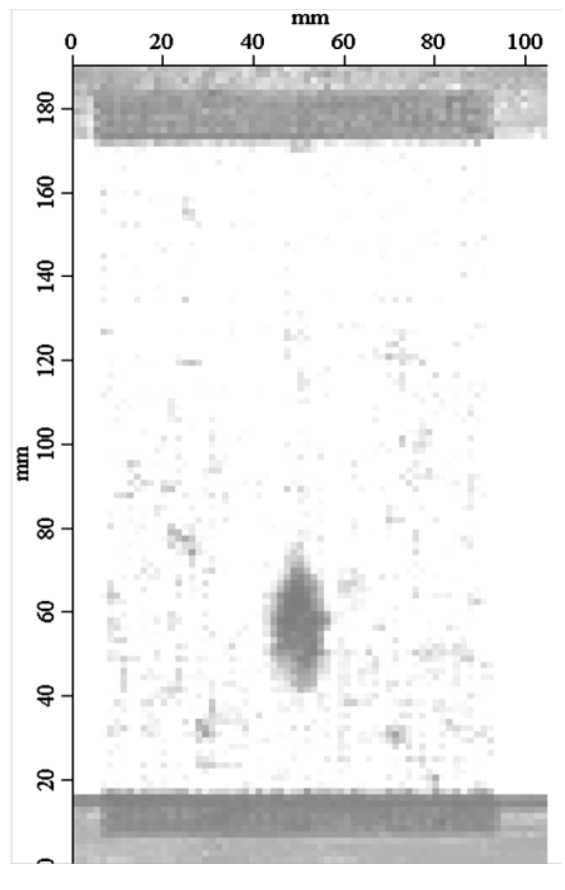

(c) 
Figure 8: EIT reconstructions of a single-ply E-Glass/EPON 862/CNT composite following an impact of (a) $10 \mathrm{~J}$ and (b) $20 \mathrm{~J}$. The hole location is represented by a black circle. The resistance damage center is marked by a blue ' $o$ '. The EIT damage center is marked by a red ' + '. The color bars correspond to the plots in their respective columns.

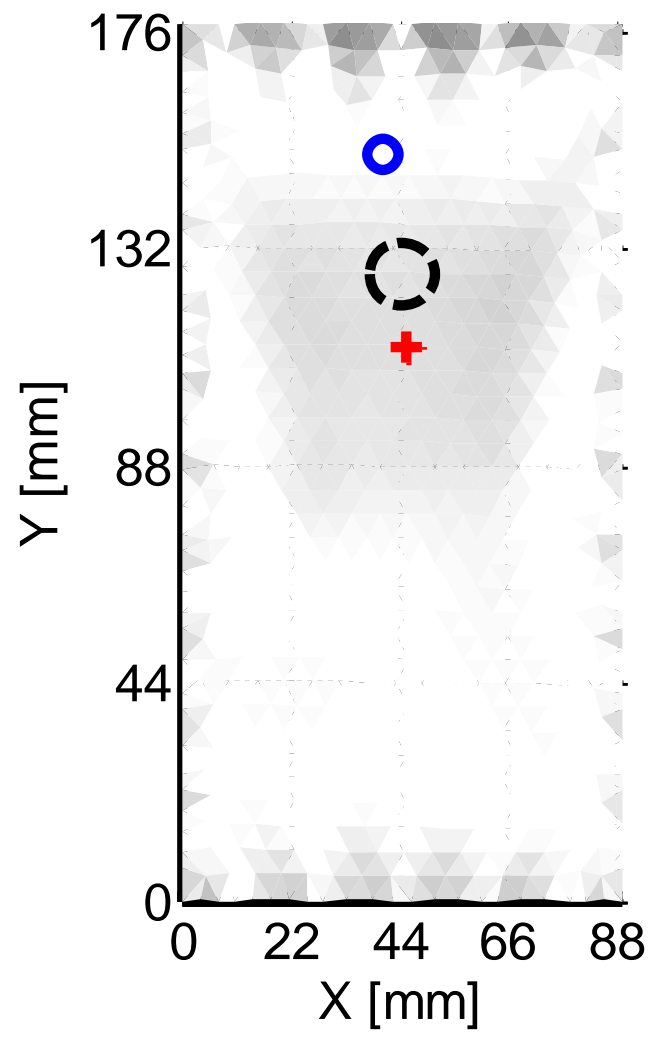

(a)

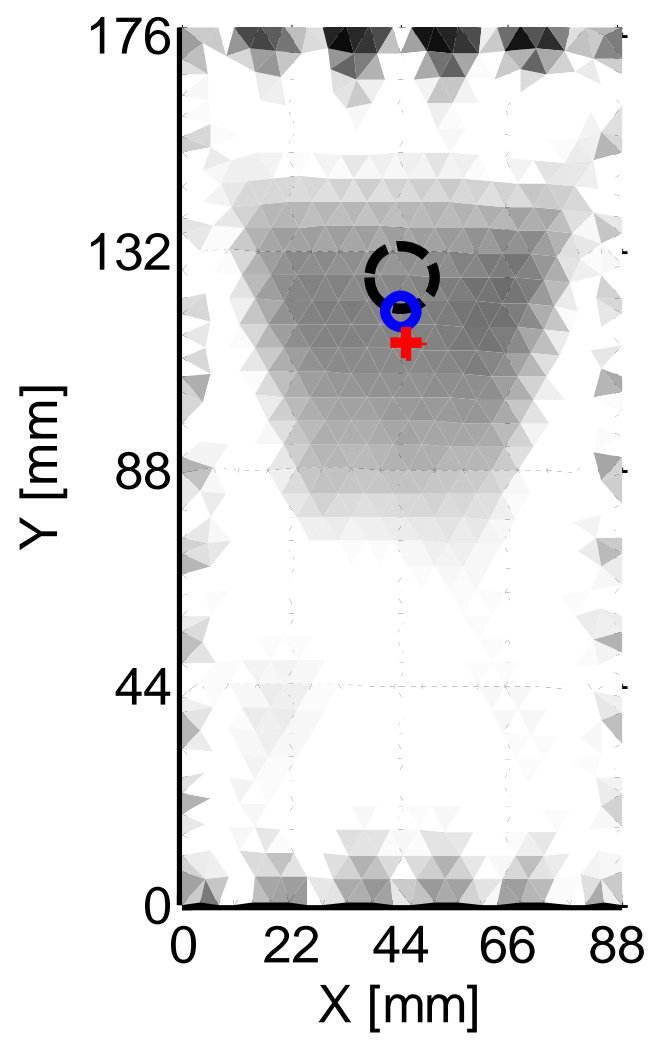

(b) 


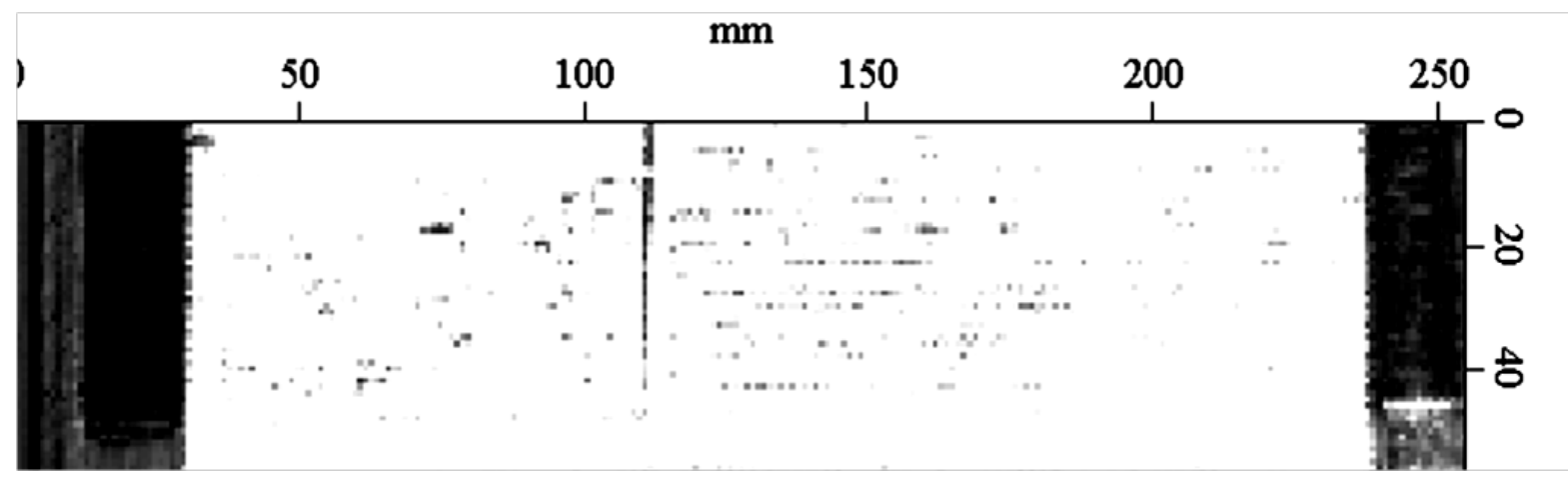

Figure 9: C-scan of [0] $]_{5}$ E-Glass/EPON 862/CNT panel with the center ply cut in order to initiate delamination. 
Figure 10 - Resistance Measurements - Delamination

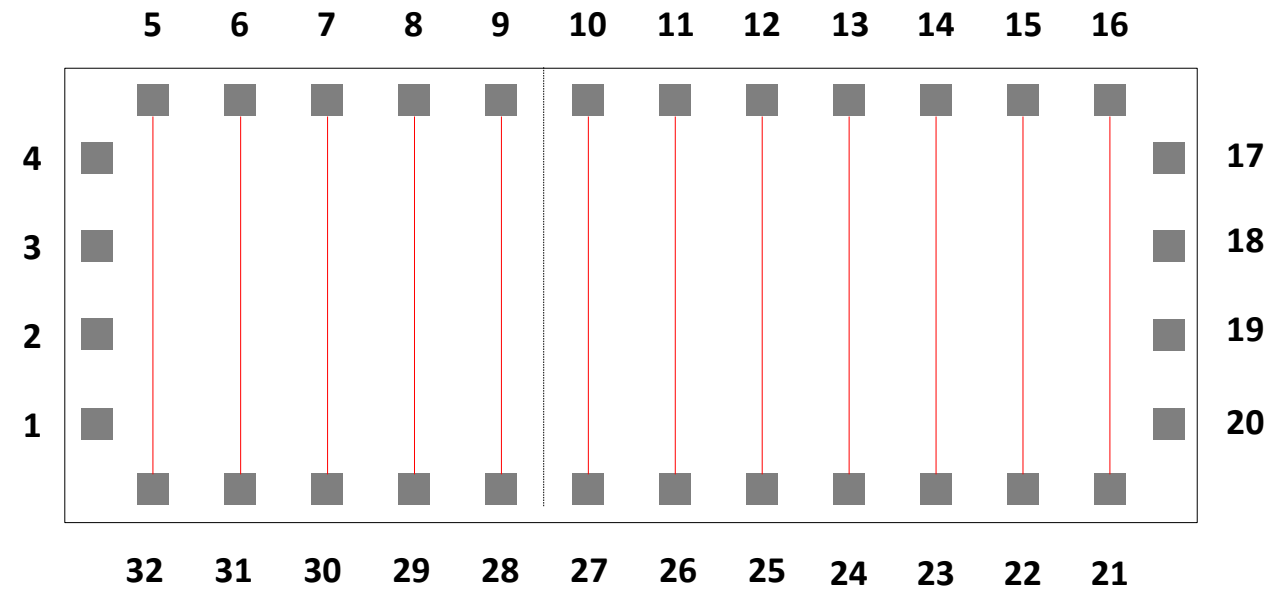

(a)

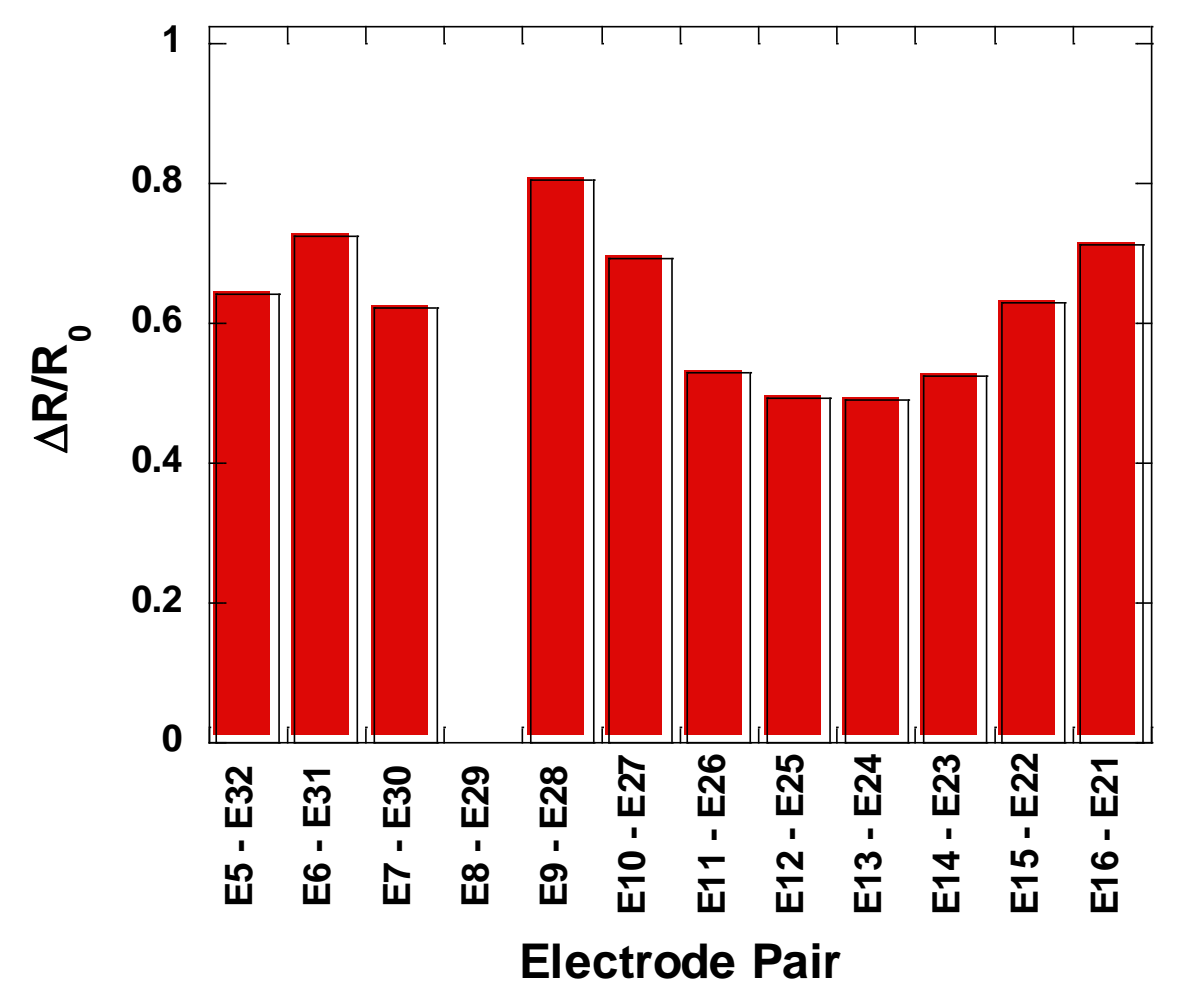

Figure 10: (a) Illu injection line: measurement indicated by a resista 

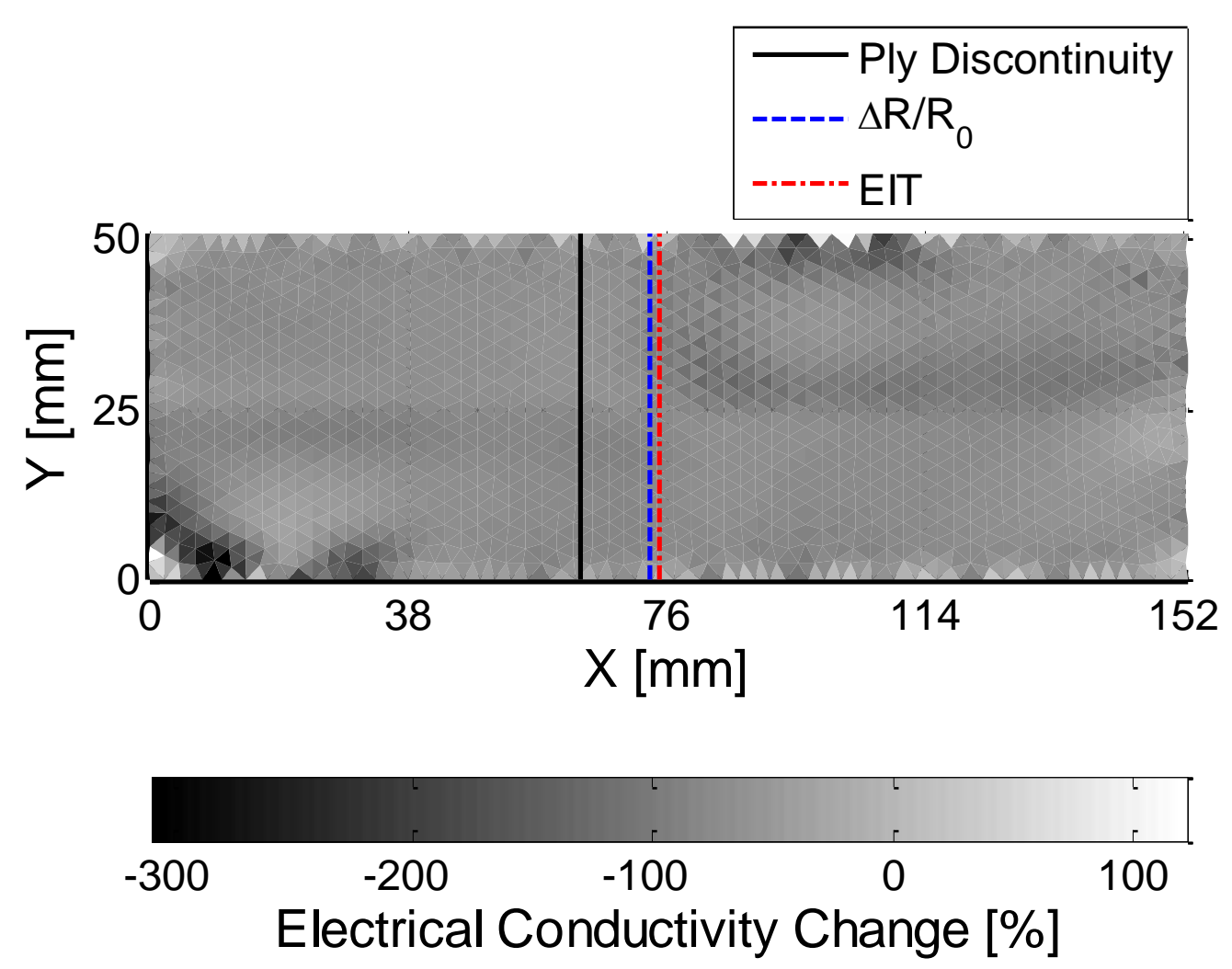

Figure 11: EIT reconstructions of the $[0]_{5} \mathrm{E}-$ Glass/EPON 862/CNT tensile bar using the across current injection pattern. 